\title{
Gas flow and dark matter in the inner parts of early-type barred galaxies ${ }^{\star}$
}

\section{SPH simulations and comparison with the observed kinematics}

\author{
I. Pérez ${ }^{1,2}$, R. Fux ${ }^{2,3}$, and K. Freeman ${ }^{2}$ \\ 1 Kapteyn Astronomical Institute, University of Groningen, PO Box 800, 9700 AV Groningen, The Netherlands \\ e-mail: isa@astro.rug.nl \\ 2 RSAA, Mt. Stromlo and Siding Spr. Obs., Private Bag, Woden PO, Canberra, ACT 2606, Australia \\ ${ }^{3}$ Geneva Observatory, Ch. des Maillettes 51, 1290 Sauverny, Switzerland
}

Received 25 February 2004 / Accepted 17 May 2004

\begin{abstract}
This paper presents the dynamical simulations run in the potential derived from the light distribution of 5 late-type barred spiral galaxies (IC 5186, NGC 5728, NGC 7267, NGC 7483 and NGC 5505). The aim is to determine whether the mass distribution together with the hydrodynamical simulations can reproduce the observed line-of-sight velocity curves and the gas morphology in the inner regions of these barred galaxies. The light distribution is obtained from the $H$-band and the $I$-band combined. The $M / L$ is determined using population synthesis models. The observations and the methodology of the mass distribution modelling are presented in a companion paper. The SPH models using the stellar mass models obtained directly from the $H$-band light distributions give a good representation of the gas distribution and dynamics of the modelled galaxies, supporting the maximum disk assumption. This result indicates that the gravitational field in the inner region is mostly provided by the stellar luminous component. When $40 \%$ of the total mass is transferred to an axisymmetric dark halo, the modelled kinematics clearly depart from the observed kinematics, whereas the departures are negligible for dark mass halos of $5 \%$ and $20 \%$ of the total mass. This result sets a lower limit for the contribution of the luminous component of about $80 \%$, which is in agreement with the maximum disk definition of the stellar mass contribution to the rotation curve (about $85 \% \pm 10$ ). This result is in agreement with the results found by Weiner et al. (2001) for NGC 4123 using a similar methodology. For two galaxies, NGC 7483 and IC 5186, a very good agreement with the observed data is found. In these cases the non-circular motions can help to break the disk-halo degeneracy. For the other three galaxies (NGC 5728, NGC 7267 and NGC 5505) no definite results are found: for NGC 7267 and NGC 5505 no steady state is reached in the simulations and for NGC 5728 there is no good agreement with the observed kinematics, possibly due to the presence of a secondary bar decoupled from the primary. However, for this latter galaxy the $M / L$ ratio used gives the right amplitude of the rotation curve, in further support of the $M / L$ calculation method used throughout this work. Fast bars give the best fit to the observed kinematics for NGC 7483 and IC 5186 with corotation at the end of the bar for NGC 7483 and at $1.4 \times R_{\mathrm{bar}}$ for IC 5186. For NGC 5505 for which no steady state configuration is found, the addition of a rigid halo stabilises the gas flows but the derived kinematics does not fit well the observations.
\end{abstract}

Key words. galaxies: kinematics and dynamics - galaxies: structure - cosmology: dark matter

\section{Introduction}

Flat rotation curves offer the most direct evidence for the existence of dark matter in galaxies. The simplest interpretation is that spiral galaxies possess massive dark halos that extend to larger radii than the optical disks. However, to get this flat rotation curve both components need to be coupled in some way. This coupling leads to a degenerate problem; the velocity field of an axisymmetric galaxy does not make it possible to uniquely disentangle the contribution of the halo from

^ Figures ??-??, ??, ??, ??, ??, ??, ??, ?? and ?? are only available in electronic form at http://www. edpsciences.org that of the disk. This disk-halo degeneracy requires a further constraint, usually the assumption of the "maximum disk". A galaxy is described as having a "maximum disk" when the stellar disk accounts for most of the rotational support of the galaxy in the inner parts. A quantitative definition for the maximum disk hypothesis is that the stellar disk provides $85 \% \pm 10 \%$ of the total rotational support of the galaxy at $R_{\text {disk }}=2.2 h_{\mathrm{R}}$, where $h_{\mathrm{R}}$ is the disk scale-length (Sackett 1997). The upper boundary avoids hollow cores in the dark halo. There is no consensus yet on whether maximum disks are preferred (Freeman 1992; Courteau \& Rix 1996; Salucci \& Persic 1999). The match to the overall shape of the rotation curve is often 
met just as well by pure halo or pure disk models. It is not even clear whether the Milky Way favours a maximum disk or not.

Gas dynamics within bars can help to break this degeneracy. The non-circular velocities that characterise gas streaming motions in a bar will allow us to determine the necessity for a dark halo in the inner parts of spiral galaxies. It has been argued that a heavy halo will slow down a bar by dynamical friction (Weinberg 1985; Debattista \& Sellwood 1998); therefore the bars embedded in a heavy halo would have to be slow rotators. However, the few directly measured pattern speeds of bars seem to indicate that they are fast rotators, implying the non existence of a dominant halo in the inner parts. Tremaine \& Ostriker (1999) explain the fact that the bars rotate fast by arguing that the angular momentum transfer from the disk to the halo would spin the latter up, flattening the inner halo. Since bars are coupled to the halos they should also rotate rapidly. In this way one obtains a fast bar embedded in a dark halo; see also Athanassoula (2002) for bar-halo coupling results.

To test whether the luminous mass in the inner parts of spiral galaxies can account for their observed gas kinematics or whether a more axisymmetric dark matter component is required, the inner gas dynamics of a sample of barred spiral galaxies has been modelled by running a $3 \mathrm{D}$ composite $N$-body/hydrocode. The complicated shapes of barred galaxies have led us to a non-analytical approach to the modelling of the gas dynamics in these systems and the potential is calculated directly from the light distribution. This approach takes into account the non-axisymmetric structures, such as bars, that are very important in the dynamics of the inner region. Some similar previous work can be found in Weiner et al. (2001).

Section 2 briefly presents the observation, data reduction and calibration carried out. In Sect. 3 the code used for modelling the gas flow will be presented. How the mass distribution is obtained from the composite $H$ and $I$ band light distribution is explained in Sect. 4.1. The initial conditions for the simulations will be presented in Sect. 4.2. The parameter space explored in the simulations is presented in Sect. 4.3. In Sect. 4.4 the analysis methods used to compare the modelled and observed line-of-sight velocity curves will be introduced. In Sects. 5 and 5.1 the results for IC 5186 are presented. This section also presents simulations within the potential derived from the $I$-band alone and compares them to those based on the composite $(H+I)$-band image (Sect. 5.2). In Sect. 5.3 the comparison for IC 5186 for different scale-heights is presented. In Sect. 6 the results for NGC 5728 are shown. Section 7 presents the results for NGC 7267. Section 8 shows the results for NGC 7483. Section 9 presents the results for NGC 5505. Section 10 presents the results of the modelling with a dark halo component, which is done for IC 5186 and NGC 5505. A discussion is given in Sects. 11 and 12 concludes the paper.

\section{Data}

The modelled galaxies form part of a larger sample of 27 isolated spiral galaxies. The whole data set, data analysis and sample characterization is presented in Pérez et al. (in prep., hereafter "data" paper) and Chap. 2 of Pérez (2003).

\subsection{Optical rotation curves}

The rotation curves were obtained by optical spectroscopic $(\mathrm{H} \alpha)$ observations along several position angles using the Cassegrain Boller \& Chivens spectrograph on the $1.52 \mathrm{~m}$ telescope at La Silla Observatory and the Double Beam Spectrograph (DBS) on the $2.3 \mathrm{~m}$ telescope at Siding Spring Observatory (SSO). At La Silla the detector was a $2 \mathrm{~K} \times 2 \mathrm{~K}$ Loral-Lesser CCD, and the dispersion was $1.0 \AA^{\mathrm{A}} \mathrm{pixel}^{-1}$ (grating \#26). The pixel scale of the CCD is $0.82 \operatorname{arcsec}_{\text {pixel }}^{-1}$, the wavelength range studied was $\lambda$ 4900-5700 $\AA$. The detector at SSO was a SITe $1752 \times 532 \mathrm{CCD}$. The gratings used were the $1200 \mathrm{~B}$ and $1200 \mathrm{R}$ for the blue and red arms respectively, giving a dispersion of $1.13 \AA$ pixel $^{-1}$ for the blue arm and $1.09 \AA \mathrm{pixel}^{-1}$ for the red arm in the wavelength interval from 4315-5283 $\AA$ and 6020-6976 A respectively. In both cases the slit was visually aligned with the galaxy nucleus at different position angles (PA) and the slit width was set to 1.5 arcsec on the sky, giving a projected width of 2.2 pixels.

Total integration times for the galaxies varied from 1 to $3 \mathrm{~h}$. In the case of the DBS the exposures had to be shorter than 20 min due to flexure of the instrument. The observations were split in three or more exposures per galaxy to eliminate cosmic rays from the frames.

All the spectra obtained were reduced using standard IRAF routines. Overscan and bias were subtracted. No dark subtraction was done due to the low dark current of the chips used $\left(1 \mathrm{e}^{-} \mathrm{pix}^{-1} \mathrm{~h}^{-1}\right)$. At the $1.52 \mathrm{~m}$ telescope, dome and sky flats were acquired in order to correct properly for slit width variation. Flatfielding correction was achieved to the $2 \%$ level. Geometrical corrections were applied to the frames in order to correct for any misalignment. The data reduction for the DBS spectra was carried out in a similar way but, due to the double beam nature of the spectrograph, care had to be taken to deconvolve the dichroic using spectrophotometric standards.

The ionised gas rotation curve and the velocity dispersions were obtained by fitting a Gaussian to the $\mathrm{H} \alpha$ and [NII] ( $26583.4 \AA)$ lines using the MIDAS package ALICE. These lines were clearly detected in all the galaxies observed. The gas rotation curves and the velocity dispersion profile derived independently from $\mathrm{H} \alpha$ and [NII] are in good agreement at all radii.

\subsection{Optical imaging}

Optical imaging observations were performed at the Swope 40 inch telescope at Las Campanas Observatory (LCO) and the 40 inch telescope at SSO. The observations at the Swope telescope were taken with a SITe 3 chip with a pixel scale of $0.435 \operatorname{arcsec}_{\text {pixel }}^{-1}$ and a field of view of 12 arcmin. The observations at SSO were taken with the direct imager and the SITe $2 \mathrm{~K}$ detector with 0.6 arcsec pixel ${ }^{-1} . B, V$ and $I$ filters were used, and three images were taken per object per band, except in some cases where only two frames were obtained. Three standard fields (Landolt 1992) were acquired per night. A series of twilight sky flats were obtained each night.

The optical data were reduced using standard IRAF routines. Bias and overscan subtraction were performed. The bias 
levels were very stable during the runs. For the SSO observations the CCDs had to be reinitialised during the night to maintain stable and structure-free bias frames.

Calibration for the photometric nights was done using APPHOT on the Landolt fields and then using PHOTCAL to fit the zero-point magnitudes, the colour and the extinction coefficients.

\subsection{Near-Infrared imaging}

Near-Infrared (NIR) photometry gives a good representation of the old stellar population and therefore of the stellar mass distribution. Furthermore, IR bands are less affected by the internal absorption of the galaxy.

NIR imaging data were obtained at LCO, using the DuPont 100 inch telescope and the Swope 40 inch telescope, and the $2.3 \mathrm{~m}$ telescope at SSO. The NIR observations at the DuPont were obtained with the IR camera with a Rockwell NICMOS3 HgCdTe $256 \times 256$ array with 0.42 arcsec pixel $^{-1}$ (1.8 $\operatorname{arcmin} \times 1.8$ arcmin field of view). The IR observations at the Swope telescope were taken with the IR camera with a NICMOS $3 \mathrm{HgCdTe} 256 \times 256$ array with a scale of 0.599 arcsec pixel ${ }^{-1}(2.5 \operatorname{arcmin} \times 2.5$ arcmin field of view $)$. CASPIR, the imager at the $2.3 \mathrm{~m}$ telescope at SSO, uses a Santa Barbara Research Center $256 \times 256$ InSb detector array, with a focal plane scale of 0.5 arcsec pixel ${ }^{-1}$. The filters used were $J, H, K_{\mathrm{n}}$ for the imaging with CASPIR and $J_{\text {short }}, H, K_{\text {short }}$ for the LCO runs. The NICMOS3 detector becomes nonlinear when the total counts (sky + object) exceed 17000 ADU. For this reason and because of the sky variability in the IR, we exposed for $60-120 \mathrm{~s}$ in $J 30 \mathrm{~s}$ in $H$ and $15 \mathrm{~s}$ in $K$. Each object was observed at several positions on the array.

For the NIR imaging obtained at LCO only the smallest angular size galaxies of the sample were observed. Therefore no offset to different sky positions was made and the sky was simply calculated by averaging a number of the stacked images for a given object with a $\sigma$ clipping rejection algorithm.

The objects measured with CASPIR were recorded at several positions on the array with a dither pattern, and larger offsets were applied to obtain sky frames with the same exposure time as the object frames. The dithering between frames was typically 20 arcsec and the offset for the sky frames was typically 2.5 arcmin. We obtained sky frames after each second object exposure, beginning and ending each exposure sequence with a sky exposure. Each single exposure time was the same as for the LCO runs.

The NIR data were reduced using standard IRAF routines and eclipse (IR data reduction package developed at ESO). All the frames had to be corrected for instrumental effects such as nonlinearity, dark current and pixel-to-pixel variation. The response of the CASPIR detector has a quadratic nonlinearity which is corrected after bias subtraction but before dark correction and flatfielding.

The HST standards list (Persson et al. 1998) and the IRIS standard star list (Carter 1995) were used. 6 to 7 standard star observations were observed per night, whenever possible repeating each standard up to 3 times. Photometric calibration was carried out in a similar way to the optical imaging calibration.

\section{Introduction to the code}

The $N$-body and hydro code was initially developed by the Geneva Observatory galactic dynamics group for spiral galaxy studies (Pfenniger \& Friedli 1993; Fux 1997, 1999). This code has been modified by Fux for the purpose of the present work. The main difference with the original code used by Fux in his thesis (Fux 1997) is that his simulations were completely selfconsistent; here the stellar potential is fixed using the observed light distribution. Only a single grid was used for the potential and force calculations, since there was no addition of a vertically extended halo. What follows is a brief summary of the code characteristics. For more details about the code, refer to Fux (1999) or his Ph.D. Thesis, Fux (1997).

The code uses the particle-mesh technique to assign to each cell of a fixed grid a mass that is proportional to the enclosed number of particles in that cell, according to the Cloud-In-Cell method (CIC). Then the potential at each of the grid points is calculated from the masses at all grid points. In our simulations the rigid potential is computed only once, assigning a Monte Carlo realisation of the mass distribution derived from the galaxy images to the grid. The CIC method is a method to linearly interpolate a discretely sampled field where each particle is represented by a "cloud" with the same volume as that of the grid cell and the mass is split up between the eight cells the cloud can cover. Fractions of that "particle-cloud" mass are then assigned to the grid points and the sum over all the particles gives the mass per cell. The potential is then calculated by Fourier transforming the density and the adopted kernel, multiplying both and then inverse Fourier transform in the $z$ and $\phi$ dimension to recover the potential (in the $R$ dimension, the potential does not appear as a convolution and therefore a direct summation is performed). The geometry chosen is a cylindrical-polar grid and the short range forces are softened using a variable homogeneous ellipsoid kernel with principal axes matched to the local grid resolution in each dimension. The polar grid gives a better radial resolution at the central parts of the disk where the density of matter is higher and the rotation period shorter.

The pressure and viscous forces are derived by 3D Smooth Particle Hydrodynamics, SPH (Benz 1990; Fux 1999). In all the models the gas is taken to be isothermal with a sound speed of $10 \mathrm{~km} \mathrm{~s}^{-1}$ and an adiabatic index of $5 / 3$, corresponding to the atomic hydrogen.

The gas particle positions and velocities are advanced by integrating the equations of motion using an integrator similar to the leap-frog algorithm but evaluating the phase-space coordinates at the same times. The code uses an adaptative time step. After each integration step, one evaluates the maximum relative contribution per unit time of the second order terms to the integrated magnitudes. If this maximum contribution exceeds a given tolerance, then the code restores the old values and integrates again with a smaller time step. For a constant time step, the integrator is time-reversible and the precision 
remains close to that of the leap-frog algorithm. The adaptative time step is very useful for resolving the shocks in the gas.

\section{Simulations}

The strategy in running the simulations was the following: first, low resolution simulations were run for all the galaxies. This was to optimise the different parameters, such as the onset time for the non-axisymmetric component, the vertical grid size, the total integration time and also whether running the code with no gas self-gravity, i.e. considering the gas as test particles, makes a significant difference to the final results. These simulations were run at the Research School of Astronomy and Astrophysics (RSAA, Canberra, Australia) to ensure no waste of time when running the high resolution simulations at the supercomputer (see below).

The size of the grid for these low resolution simulations is 31 cells in the radial direction, 32 in the tangential direction and 242 (including doubling up cells) in the vertical direction with a total of 50000 particles. The rotation direction of the bar is chosen to be opposite to the winding of the spiral arms outside the bar. Only two pattern speeds were checked (placing corotation (CR) at the end of the bar and at twice the semi-major axis of the bar) and six onset times for the nonaxisymmetric component were tried, with the bar fully grown at $0.5,1.0,2.0,3.0,4.0$ and 5.0 bar rotations. These simulations were carried out to ensure that at the chosen onset time the particle flow adjusted steadily (see also Sect. 4.2).

The high-resolution simulations were run at the Australian National University (ANU) supercomputer facility using the Alpha Server SC system. Several models were run using $H$ and $I$ photometry for the different galaxies to explore the parameter space of the bar pattern speed in order to reproduce the observed position-velocity diagrams. Then, for IC 5186 several runs with the best fit bar pattern speed were carried out but this time using for the mass distribution only the $I$-band instead of the composite $(H+I)$-band image. This was done to test the effect of using a band that is more affected by extinction. The results of this test are presented in Sect. 5.2.

To check whether the observed kinematics could be reproduced with an additional axisymmetric component, we ran simulations with a dark halo component on two of the five galaxies (Sect. 10). In practice, this is done in the following way. When growing the bar in the beginning of a simulation, the gravitational potential is decomposed into

$\Phi=f \Phi_{\text {full }}+(1-f) \Phi_{\text {axisym }}$,

where $\Phi_{\text {full }}$ is the full non-axisymmetric potential as derived from the population synthesis models, $\Phi_{\text {axisym }}$ its azimuthal average, and $f$ a factor that increases linearly with time from 0 to 1 during the onset time. If one stops the growth of the bar at a percentage $f$ of that onset time and then carries on the simulation maintaining this partly grown bar, the effect is such as to replace a fraction $(1-f)$ of the inferred visible mass by an axisymmetric component, which we consider here as our dark matter halo. In this way, we reduce the azimuthal forces while keeping the same average circular velocity. Furthermore, this method, which reduces the strength of the bar while leaving the total mass and the axisymmetric part of the rotation curve unchanged, was very easy to implement in the code. The flatness of this dark halo it is not a crucial problem in the modelling because the gas dynamics do not depend much on the vertical forces.

The gas flow in the inner parts will be influenced by the choice of the vertical scale-height, with a higher scale-height giving effectively a smoother potential. Tests with different scale-heights were carried out for IC 5186 to investigate how the computed kinematics are affected (see Sect. 5.3).

The high-resolution simulations were run with the following characteristics: grid size used is 95 cells in the radial, 96 in the azimuthal and 1214 (including doubling up) in the vertical directions. The vertical resolution is set to 0.05 times the scaleheight adopted for the luminous mass distribution. The number of gas particles used is 300000 . The barred potential rotates at fixed pattern speed $\Omega_{\mathrm{b}}$. Low-resolution simulations show gas self-gravity to be irrelevant for the results so we finally ran the code with no self-gravity.

\subsection{Mass distribution}

The gravitational field is calculated from a surface density distribution that follows the light distribution of the combined $H$ and $I$-band images of the image projected to face-on, as described in Pérez et al. (in prep., hereafter Paper II) and Chaps. 3 and 4 of Pérez (2003). The $M / L$ ratio is fixed using stellar population synthesis models as described also in Paper II. In this way, we also test the reliability of the population synthesis $M / L$ ratios. In a manner consistent with previous studies (de Jong 1996), where colour gradients in the disks of spiral galaxies are found, 2D $M / L$ ratio maps taking into account these colour changes were built, assuming that they are mainly due to population changes. In the process of calculating the $M / L$ ratios it is clear that the main factor affecting the values obtained is the Initial Mass Function (IMF). We adopted a Salpeter IMF with a flatter slope for $m<0.5 M_{\odot}$. Though one might think that this is a degenerate problem where we cannot distinguish between a scenario with a different IMF and that of a sub-maximum disk, in fact the $M / L$ adopted is not just a scale factor since we are assuming a $2 \mathrm{D} M / L$ map and we are not only looking at the maximum of the rotation curve, but also at the shape of the line-of-sight (L-O-S) velocity curves.

For the deprojection of the galaxies a flat disk was adopted and a simple geometrical deprojection was applied. An analytical model was chosen for the extinction. In this model the stars lie above and below the layer of the gas and dust. A radially exponential varying optical depth was added to the model. The methodology followed is described in Paper II.

For the vertical distribution an exponential profile is assumed with a radially constant vertical scale-height. This may not be a good assumption as there is evidence from infrared photometric studies of the Milky Way that the vertical scale of the bar is larger than that of the disk (Freudenreich 1998). The biggest impact on the dynamics of a non-constant scaleheight is precisely in the inner region we are interested in, where the radial forces will change significantly. However, 
Table 1. HI gas and stellar masses for the modelled galaxies.

\begin{tabular}{lcc}
\hline \hline Name & HI mass in $M_{\odot}$ & Stellar mass in $M_{\odot}$ \\
\hline IC 5186 & $9.36 \times 10^{9}$ & $4.94 \times 10^{10}$ \\
NGC 7483 & $6.49 \times 10^{9}$ & $2.37 \times 10^{11}$ \\
NGC 5505 & $2.01 \times 10^{9}$ & $1.16 \times 10^{11}$ \\
NGC 5728 & $2.5 \times 10^{9}$ & $4.70 \times 10^{11}$ \\
NGC 7267 & $1.00 \times 10^{9}$ & $6.86 \times 10^{10}$ \\
\hline
\end{tabular}

it is hard to do better than this since not much is known about the scale-heights of bars in external galaxies. For most of the simulations one value of the scale-height was adopted, following the relationship found by Kregel et al. (2002). They analysed the structure of the stellar disk in a sample of edge-on galaxies and found that the average $\left\langle h_{R} / h_{z}\right\rangle=7.3 \pm 2.2$, where $h_{R}$ and $h_{z}$ are respectively the disk exponential scale-length and scale-height. They also found an average $R_{\max } / h_{R}=3.6 \pm 0.6$, where $R_{\max }$ represents the disk truncation radius. The scalelengths are derived from the $H$-band images. The different values of the scale-height and the scale-length in the simulations are presented in Table 2. Table 1 presents the gas and stellar masses for reference although the gas mass is not included in the potential of the simulations because we do not know its distribution. The gas mass represents the HI mass; no information on $\mathrm{CO}$ is found in the literature for most of the galaxies, except for NGC 5728 (Combes \& Leon 2002) where the CO mass represents $3.0 \times 10^{9} M_{\odot}$, comparable to the HI mass. Even when the $\mathrm{CO}$ mass is added to the HI mass the total gas mass still represents only a small fraction of the total stellar mass.

\subsection{Initial conditions}

The initial gas density in the simulation consists of one component. The radial distribution for the gas is a Beta function with a standard deviation set to the scale-length of the visible disk (Table 2) and radially vanishing at a distance 4 times this scale-length. The vertical distribution of the gas is generated directly by solving the hydrostatic equilibrium equation for an isothermal gas. Different initial density distributions have little effect on the results. The gas particles have pure circular motion with cylindrical rotation and zero velocity dispersion, since the effective dispersion is taken into account in the pressure component of the SPH.

Based on the low resolution simulations, the bar is chosen to grow linearly during three bar rotations, so that the gas flow can steadily adjust to the forcing bar without requiring to much CPU time. For growing times shorter than this the flow took some more time to settle, depending on the galaxy, and of course always reached the same final state. However, if one chooses a shorter bar growth time and does not run the simulations long enough one could be seeing misleading transient features and interpret them as part of the steady flow.
Table 2. Exponential scale-heights and scale-lengths, distances and grid sizes for the modelled galaxies.

\begin{tabular}{lcccc}
\hline \hline Name & $\begin{array}{c}\text { Distance } \\
(\mathrm{Mpc})\end{array}$ & $\begin{array}{c}h_{R} \\
(\mathrm{kpc})\end{array}$ & $\begin{array}{c}h_{z} \\
(\mathrm{kpc})\end{array}$ & $\begin{array}{c}\text { Grid extent } \\
(\mathrm{kpc})\end{array}$ \\
\hline IC 5186 & 65.57 & 4.591 & 0.629 & 55.0 \\
NGC 7483 & 65.85 & 11.00 & 1.507 & 104.0 \\
NGC 5505 & 57.13 & 1.897 & 0.260 & 29.0 \\
NGC 5728 & 37.17 & 5.451 & 0.747 & 90.0 \\
NGC 7267 & 44.70 & 2.503 & 0.343 & 41.0 \\
\hline
\end{tabular}

\subsection{Model parameters}

We made a series of standard runs for each galaxy, and then some exploratory runs to test the effect of varying different parameters in the simulations. The standard runs were run with fixed stellar $M / L$ ratio and fixed constant scale-height. Several bar parameters have a large effect on the gas response: the central concentration, the axial ratio, the mass of the bar and the pattern speed. The first three are fixed by the given potential (in the standard runs) and the only one that can be varied is the pattern speed, $\Omega_{\mathrm{b}}$. This parameter fixes the position of the resonances and controls much of the gas response.

For each galaxy 6 different pattern speeds were modelled. The $\Omega_{\mathrm{b}}$ is parametrised by the ratio of the corotation radius assuming axisymmetry to the bar semi-major axis $R_{\mathrm{CR}} / R_{\mathrm{bar}}$. Table 5 gives the pattern speeds modelled for the different galaxies. The bar radius has been obtained as explained in the data paper.

For the test runs the pattern speed was fixed to that giving the best model (see Sect. 4.4 for an explanation of the criteria) in the standard runs and other parameters that could affect the gas response were explored. The scale-height of the stellar component also has an effect on the dynamics of the gas in the inner parts. Larger scale-heights smooth out the potential, reducing the radial gas flows. For IC 5186, the scale-height dependence of the velocity flow was investigated. Models were run with three different scale heights at three pattern speeds. The scale-heights explored were $0.5,1.0$ and 1.5 times the one used in the standard runs.

For the models with a rigid halo (IC 5186 and NGC 5505): percentages of 5, 20 and 40 of the total mass ascribed to the dark halo were tested in the manner explained in Sect. 4.

\subsection{The best fit models}

The best fit model requires in the first place that the model slit position-velocity diagrams best reproduce the observed rotation curve with the following criteria. The position-velocity diagrams are extracted from the models for comparison with the long-slit observations. The velocities are projected on the plane of the sky, giving the model the same inclination as the real galaxy. Then a virtual slit is placed on the model and the position-velocity diagram is extracted at the observed position angle. The kinematics of the SPH particles are not convolved with the SPH kernel; however, this does not affect the 
derivation of the first velocity moments. To compare the modelled and observed position-velocity diagrams, the simulated data are linearly interpolated in position to the observed data. Then a $\chi^{2}$ comparison of the two velocity profiles is computed. When computing the $\chi^{2}$ a velocity dispersion component of $10 \mathrm{~km} \mathrm{~s}^{-1}$ is added, corresponding to the assumed sound speed in the models. Because our aim is to exploit the constraining power of the non-circular motions induced by the bar, we restrict the comparison to the bar region only, where these motions are strongest, and exclude the surrounding disk. Furthermore, we are assuming the same pattern speed for the spiral arms as for the bar; since these are non self-consistent models and the spiral arms are more likely to be transient features, it is safest to consider only the bar region for our comparison with the observed kinematics.

It is hard to compare the gas distribution of the galaxies with the modelled gas density distribution since no $2 \mathrm{D}$ information on the gas distribution for the sample galaxies exist in the literature. To get an idea of how good the modelled gas density distribution is, a comparison with the observed images is performed. The $B$-band light follows mainly the regions dominated by the young stellar population, indicating regions of recent star formation. These regions are in turn tracers of gas and dust in the galaxy. The dust lanes can be used to trace shocks in the gas flow (Athanassoula 1992) which can be compared to the modelled velocity fields. The dust lanes are correlated with high densities in the gas distribution. Visual comparison of the modelled gas distribution with the $B$-band images was performed. To emphasise the non-axisymmetric part of the gas distribution of the non-axisymmetric part a masking technique is applied to both the observed galaxies and the modelled distribution. An axisymmetric distribution of the $B$-band image was created by averaging azimuthally the flux over ellipses centered on the galaxy at fixed position angle and ellipticity and then subtracted from the original image. The same was done to the modelled density map convolved with a Gaussian to imitate the $B$-band image spatial resolution. Now we discuss the individual galaxies.

\section{IC 5186}

IC 5186 is an isolated SBab galaxy, at a distance of $65 \mathrm{Mpc}$. There is no kinematic nor photometric study of this galaxy in the literature. Optically an inner ring, a bar, and filamentary structure in the region between the bar and the ring are observed. The $K$ and $H$-band images show a much smoother distribution of the inner region (see Fig. 1). Long-slit spectra along the major axis (when speaking about the major/minor axis kinematics this always refers throughout the text to the apparent principal axes of the galaxy projected on the sky) show a steep gradient in the inner part of the rotation curve with a maximum velocity of $180 \mathrm{~km} \mathrm{~s}^{-1}$ at 2 arcsec. The minor axis shows a double component. Although it is difficult to disentangle the different components, there seems to be a velocity difference between the components of $\approx 200 \mathrm{~km} \mathrm{~s}^{-1}$ over a region of about $2.5 \mathrm{kpc}$ (data paper). The bar position angle is $\approx 45^{\circ}$ with respect to the line of nodes.
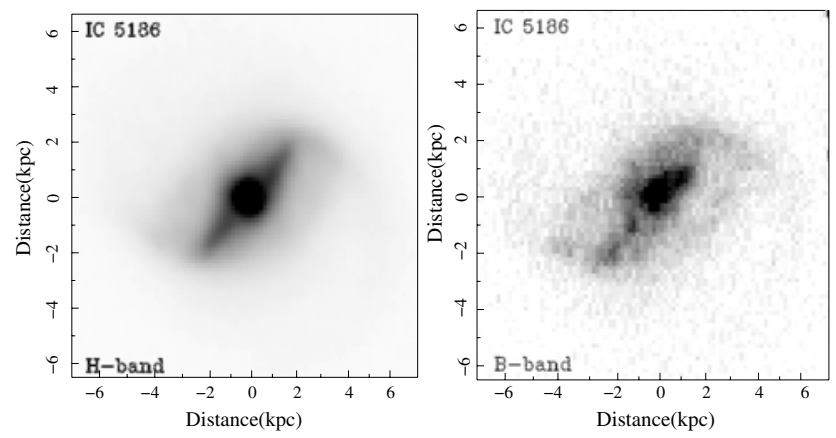

Fig. 1. Light distribution in different bands for IC 5186. The left panel shows the light distribution in the $H$-band and the right panel the light distribution in the $B$-band. Notice the smoother distribution of the light in the inner region for the $H$-band.

Table 3. Parameters for the test runs on IC 5186.

\begin{tabular}{lcc}
\hline \hline$R_{\mathrm{CR} / R_{\text {bar }}}$ & $\begin{array}{c}\text { Scale-height of } \\
\text { of stellar disk }(\mathrm{kpc})\end{array}$ & $\begin{array}{c}\text { \% of dark halo mass } \\
\text { from the total mass }\end{array}$ \\
\hline 1.0 & 0.629 & 5 \\
1.0 & 0.629 & 20 \\
1.0 & 0.629 & 40 \\
1.0 & 0.943 & 0 \\
1.0 & 0.314 & 0 \\
\hline 1.4 & 0.629 & 5 \\
1.4 & 0.629 & 20 \\
1.4 & 0.629 & 40 \\
1.4 & 0.943 & 0 \\
1.4 & 0.314 & 0 \\
\hline 1.6 & 0.629 & 5 \\
1.6 & 0.629 & 20 \\
1.6 & 0.629 & 40 \\
1.6 & 0.943 & 0 \\
1.6 & 0.314 & 0 \\
\hline
\end{tabular}

The optical emission line spectra show $[\mathrm{NII}] / \mathrm{H} \alpha \approx 1$ which could suggest shocks; however, no [OI] $(\lambda 6300 \AA)$ is present and $\log ([\mathrm{SII}] / \mathrm{H} \alpha) \approx-1.0$ (typical of HII regions). The ionisation temperature is probably low since no trace of [OIII] $\lambda 5007 \AA$ is found in any of the spectra. The observations show that $\log (\mathrm{O} / \mathrm{H})$ is high, probably higher than solar, since $\mathrm{OIII} / \mathrm{H} \beta$ is around $1 / 3$, which is consistent with abundances found in the central parts of other galaxies.

\subsection{Results}

After three bar rotations, when the non-axisymmetric component is fully grown, the gas distribution of the simulations in the potential derived from the composite $(H+I)$-band light distribution settles in a steady configuration for all the bar pattern speeds. In all cases an elongated inner ring forms parallel to the bar major axis. Inside this ring for $R_{\mathrm{CR}} / R_{\mathrm{bar}}<1.4$ a nuclear 

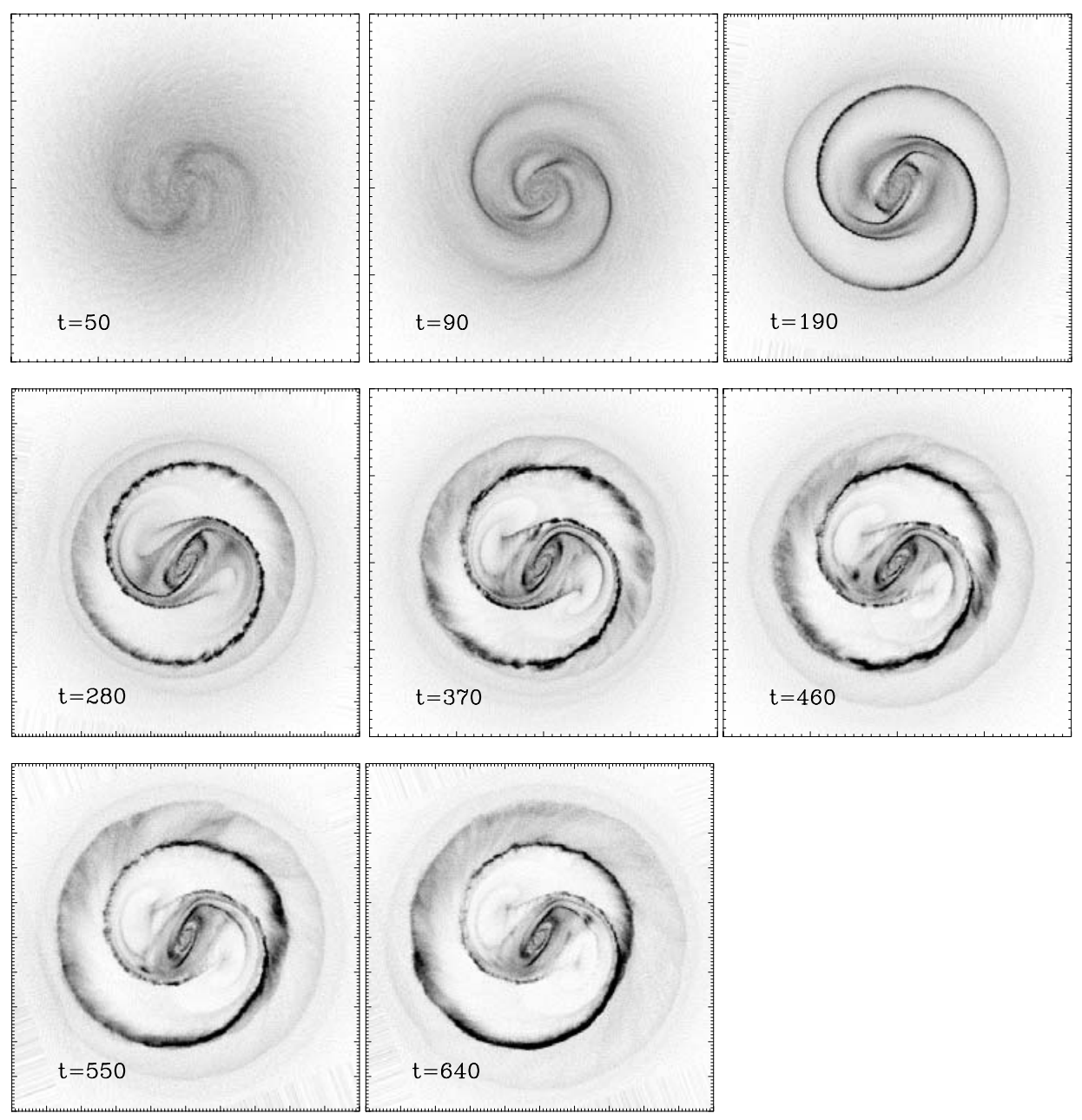

Fig. 2. Time evolution of the gas distribution in the IC 5186 simulation with corotation at $1.4 \times$ the bar semi-major axis. Each panel, from left to right and top to bottom, shows the distribution at different integration times, from 1 bar rotation (second panel) to 7 bar rotations, and in the frame corotating with the bar. The non-axisymmetric component is fully grown after three bar rotations and the bar pattern is rotating counter-clockwise in the inertial frame. The size of the frames is $20 \mathrm{kpc}$ and the time is in Myr. Notice the gas trapped around the $L_{4,5}$ points after three bar rotations.

spiral is formed but for $R_{\mathrm{CR}} / R_{\mathrm{bar}} \geq 1.4$ a nuclear ring forms with no mini spiral structure. From the frequency plot for the axisymmetric system it appears that no inner Lindblad resonaces (ILRs) are present. This nuclear ring is elongated parallel to the major axis of the bar and does not seem to be related to any resonance. To get a proper insight into the relationship of the different features with the different periodic orbits an orbit analysis is needed.

Morphologically there is little change in the gas distribution for the different pattern speeds, apart from these very central features and the fact that the features move outward as the pattern speed decreases. Figure 2 shows the time evolution of IC 5186 for a pattern speed of $\Omega_{\mathrm{b}}=63.4 \mathrm{~km} \mathrm{~s}^{-1} \mathrm{kpc}^{-1}$ $\left(R_{\mathrm{CR}} / R_{\mathrm{bar}}=1.4\right)$; each figure shows the gas density evolution after a further bar rotation. At all times, gas seems to be trapped in orbits around the $L_{4}$ and the $L_{5}$ Lagrangian points, near to the minor axis of the bar. Figure ?? shows the line-of-sight velocity curves along the major axis for models with $R_{\mathrm{CR}} / R_{\mathrm{bar}}$ of 1.0 , $1.1,1.2,1.3,1.4$ and 1.6. From this figure it is not immediately obvious which one is the best fit. However, $R_{\mathrm{CR}} / R_{\mathrm{bar}}=1.4$ looks best, supported by the $\chi^{2}$ statistic presented in Table 5 . The $\chi^{2}$ computed for IC 5186 includes only the major axis data.

For the minor axis, as already mentioned, the emission lines show very broad profiles with a hardly distinguishable double component. Therefore the position-velocity diagram is more difficult to interpret. In Fig. ?? the modelled minor axis kinematics is plotted together with the data. The central emission lines of the observed data have not been plotted due to the fact that no single line profile could be fitted. The model with corotation at 1.4 times the bar semi-major axis is consistent with the observed kinematics. The model shows highly non-circular motions on the minor axis only within the bar region, whereas in the observations the non-circular motions extend beyond the bar region.

Although we cannot directly compare the modelled and observed gas distributions, a comparison with the masked $B$-band image was carried out as explained in Sect. 4.4. One can see from Fig. ?? that the agreement in the inner regions is very good for $R_{\mathrm{CR}} / R_{\mathrm{bar}}=1.4$, except that no sign of the ring which formed in the simulation is observed in the $B$-band image. 

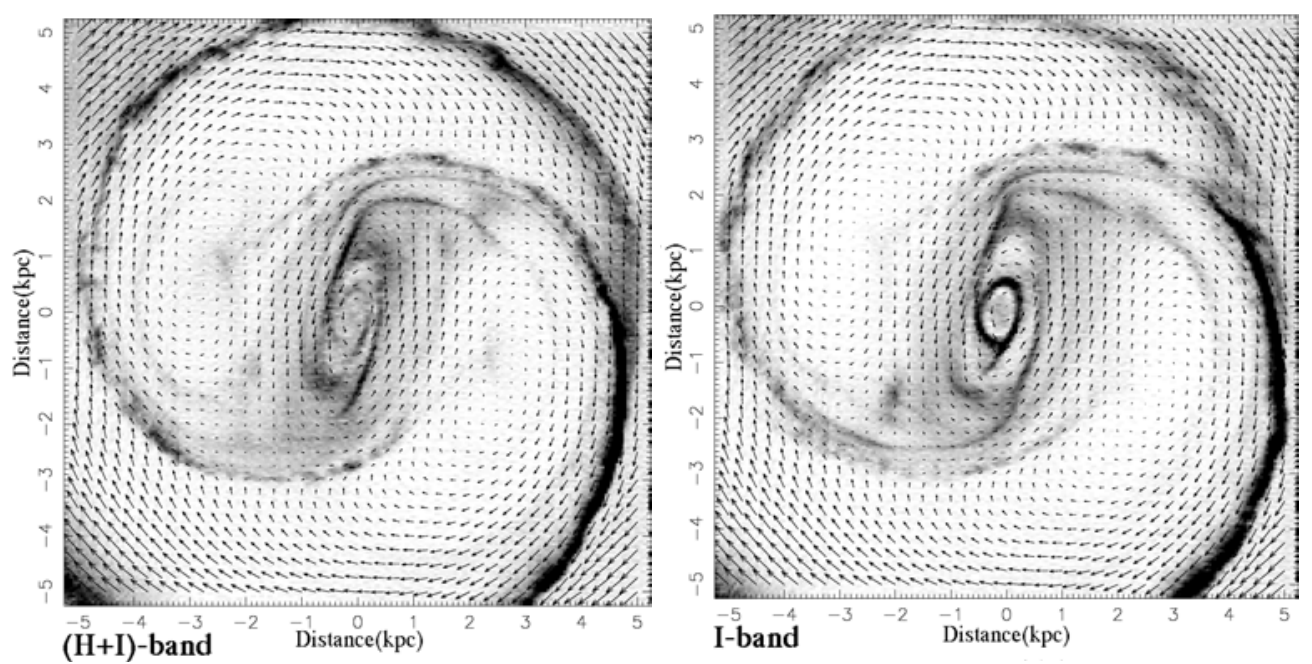

Fig. 7. The gas distribution and velocity field for the mass distributions of IC 5186 derived from the composite $(H+I)$-band image distribution (right) and the $I$-band (left panel), after 7 bar rotations and for $R_{\mathrm{CR}} / R_{\mathrm{bar}}=1.4$. The length of the vectors is proportional to the velocity in the frame rotating with the bar. Notice the clear inner ring which develops in the $I$-band simulations. The $H$-band simulation does not show this ring so clearly; however, part of the gas particles have been trapped around the $L_{4}$ and the $L_{5}$ Lagrangian points (which are the zero velocity locations near the direction perpendicular to the bar). This trapping also occurs for the $I$-band simulation but to a lesser degree.

There might no longer be gas in the inner ring. High spatial resolution imaging of the gas, maybe $\mathrm{CO}$ observations, would be needed to disentangle the morphology of the gas in the inner parts and compare it to the model gas density distribution.

When doing the same for decreasing pattern speeds, the main structural features in the model move radially outward relative to the observed structures. It seems that in order to match the main features spatially some fine-tuning of the pattern speed would be required, giving a slightly slower bar pattern speed.

\subsection{Model with mass distribution derived from the I-band light distribution}

Models were run for a mass distribution derived from the $I$-band luminosities to test the effect on the resulting gas distribution and gas kinematics of using a photometric band more affected by extinction. These models were run for three different pattern speeds, and apart from the mass distribution the other initial conditions were identical to those used to simulate the composite $(H+I)$-band image of IC 5186. The light distribution for the $H$-band is smoother than that of the $I$-band, which shows a more clumpy structure along the bar similar to that observed in the $B$-band (Fig. 1). One can also see the effect of dust in the fact that the inner ring is brighter on one side of the galaxy.

The modelled gas distribution shows a striking difference. The nuclear ring that is barely visible in the models derived from the $(H+I)$-band image is the main morphological feature in the gas distribution of the $I$-band model, see Fig. 7. In order to determine the nature of the nuclear ring the angular momentum distribution for both cases was examined (see Fig. ??). The main peak observed for both distributions corresponds to the outer ring. Most of the particles lie outside the outer Lindblad resonance (OLR), simply reflecting the extended initial gas distribution. The low angular momentum peak for the $I$-band case corresponds to the nuclear ring seen in this case but barely seen on the composite image run. For the $I$-band run there seems to be movement of particles from inside corotation to populate the innermost ring.

The inner ring seems to be related to the inner ultra harmonic resonance (IUHR), showing a four-cornered structure. The nuclear ring does not seem to be associated with any resonance and it is more likely linked to the $x 1$ family of orbits in the bar. The $H$-band models seem to have trapped more gas around the $L_{4}$ and the $L_{5}$ Lagrangian points. Figure 7 suggests that the nuclear ring more clearly present in the $I$ band case is made out of the particles that are trapped around the $L_{4}$ and the $L_{5}$ Lagrangian points and in the inner ring in the $(H+I)$-band case. The density enhancement near the Lagrangian points $L_{4,5}$ is displaced azimuthally relative to $L_{4,5}$ in both cases (see Fig. 7). The stability of the Lagrangian points could be checked to establish whether the density enhancement around the $L_{4}$ and the $L_{5}$ point is due to the fact that these points might be stable. However, the fact that the potential is not bisymmetric may complicate the derivation of the Lagrangian points. This question will be further addressed in a subsequent orbit analysis paper (in prep.). The inner ring seems to have shrunk further in the $(H+I)$-band model than in the $I$-band case, reflecting a higher degree of shock dissipation. In the axisymmetric approximation there is no ILR at this pattern speed in both cases. However, to identify properly the resonances a detailed orbit analysis is required. The position-velocity diagrams derived from the $I$-band and from the composite image do not show significant differences. To be able to conclude something about which model gives the best representation of the galaxy dynamics 2D kinematic information would be needed.

Summarising, the models derived for the mass distribution from the $I$-band alone and from the $(H+I)$-band seem to give similar overall results. There is a difference in the inner parts, 

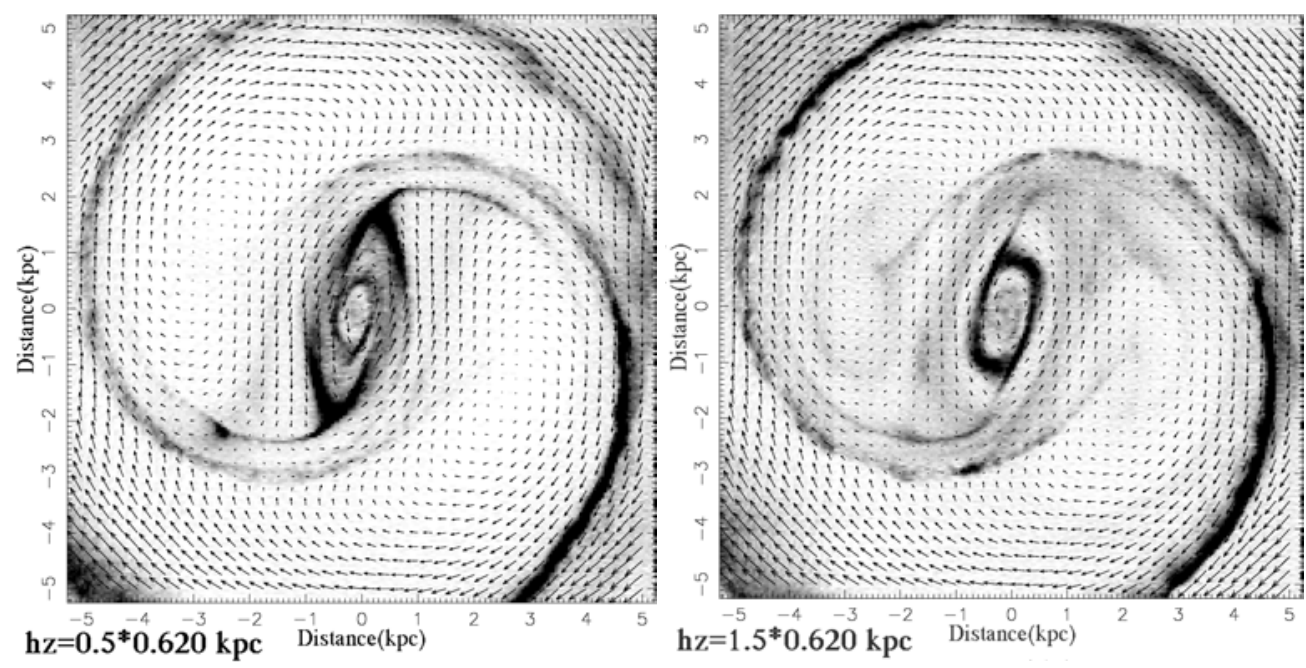

Fig. 8. The gas distribution and velocity field for the mass distribution of IC 5186 derived from the composite $(H+I)$-band image and with a scale-height of 0.5 (left panel) and 1.5 (right panel) times the adopted standard one, after 7 bar rotations and for $R_{\mathrm{CR}} / R_{\mathrm{bar}}=1.4$. The length of the vectors is proportional to the velocity in the frame rotating with the bar. The gas distributions show very different features. There seems to be more gas near the lateral Lagrangian points for a larger scale height; in fact the amount of gas there seems to be related to the softening of the potential, either via thickening the disk or by axisymmetrising it with a dark halo component, as illustrated in Fig. 23.

where the $I$-band derived model develops a clear nuclear ring whereas the $(H+I)$-band derived model develops the nuclear ring but it is not so marked. However, in this case there is a larger number of particles populating the region around the $L_{4,5}$ Lagrangian points. There are differences in the light distribution in the inner $3 \mathrm{kpc}$ region. The mass model derived from the $I$-band image presents a more massive central core than for the $(H+I)$-band mass distribution. Since the $H$-band is a better tracer of the stellar mass one might expect the opposite. However, as discussed in Paper II, the $M / L_{I}$ is more sensitive to galaxy color changes than the $M / L_{H}$. The central regions of IC 5186 are more affected by extinction in the $I$-band and therefore redder (though also dimmer), resulting in a overestimation of the $M / L_{I}$. Increasing the central mass concentration favours the nuclear ring formation, as observed in simulations by different authors (Regan \& Teuben 2003).

\subsection{Comparison of the models for different scale-heights}

The goal of this test is to check the effect of changing the scale-height in the modelled gas density distribution and the modelled position-velocity diagrams. In addition to the simulations with the adopted standard scale-height $h_{z}$, simulations with two other scale-heights were run, i.e. with $0.5 \times h_{z}(0.31 \mathrm{kpc})$ and $1.5 \times h_{z}(0.94 \mathrm{kpc})$. As one can see from Fig. 8 a change in scale-height produces a significant change in the gas distribution in the inner parts. From the velocity field one can see that the shocks for the models with lower scale-height are stronger, as one would expect since these models are enhancing the non-axisymmetry in the potential. The larger scale-height produces fewer shocks since the increase in the scale-height acts as a softening the potential. Figure ?? shows the comparison between the masked observed $B$-band and the masked models with dif- ferent stellar scale-heights. The modelled position-velocity diagrams (see Fig. ??) show a large difference with respect to the observed rotation curve for the short scale-height, and none of the pattern speeds gives good fit; for the models with $1.5 \times h_{z}$ the best fit corresponds to the pattern speed with corotation at 1.6 times the bar semi-major axis. However, a scale height of $1.5 \times h_{z}(0.94 \mathrm{kpc})$ is an unrealistically large value for the vertical scale of a real galaxy with the observed scale-length (Kregel et al. 2002).

\section{NGC 5728}

NGC 5728 is a SABa Seyfert type 2 galaxy at a distance of $35 \mathrm{Mpc}$. It shows an outer and inner stellar ring and there is also evidence for the presence of a secondary stellar bar as reported by Wozniak et al. (1995). There is no nuclear bar structure in the molecular gas, and at larger scales the $\mathrm{CO}$ emission traces the primary bar and the outer ring structure (Combes \& Leon 2002). The bar position angle is $\approx 30^{\circ}$ to the line of nodes.

The models are integrated for six bar rotations (see Fig. 12 with $R_{\mathrm{CR}} / R_{\mathrm{bar}}=1.0$ ), Table 5 shows the pattern speeds explored in the simulations. The spiral arms do not remain stationary but since the simulations are not self-consistent and we assume a single pattern speed this behaviour in the outer parts is not surprising. A nuclear ring develops with an orientation slightly tilted with respect to the inner ring (Fig. 14). The orientation of the nuclear ring with respect to the inner ring does not vary in time. This nuclear ring observed in the models has the same orientation as the secondary bar in the real galaxy (see Figs. 14 and 11). The inner ring lies very close to the corotation zone with the gas following a clear highenergy $x_{1}$-family orbital shape. The frequency diagram in the axisymmetric approximation shows that for $R_{\mathrm{CR}} / R_{\mathrm{bar}}=1$ two ILRs exist. However, the nuclear ring is not oriented perpendicular to the large scale bar and thus does not seem to be supported by $x_{2}$ orbits. If one compares the light distribution 

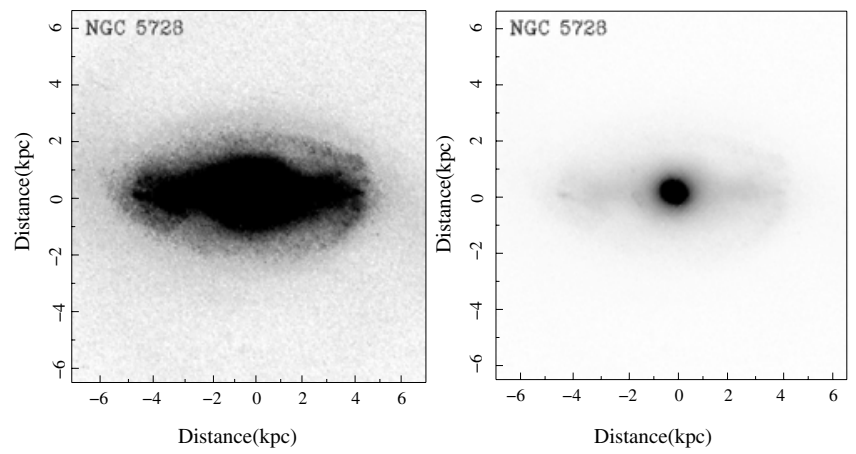

Fig. 11. Deprojected light distribution in the $H$-band of the inner part of NGC 5728. The left panel shows the extent of the primary bar and the right panel the extent of the nuclear structure.

in the $H$-band (see Fig. 11) with the gas density distribution (Fig. 14) one can observe in the $H$-band image (Fig. 11) that the gas distribution seems to coincide with the observed stellar ring. The $H$-band image suggests that the bar has the typical shape of $x_{1}$ orbits looping near the apocenter. The gas cannot stay in such intersecting orbits, therefore it is no surprise that no gas is found in the models on these lower energy $x_{1}$ orbits. However, to identify the various orbit families properly, an orbit analysis is necessary, which is beyond the scope of this study, focused on the dark matter content.

There is not a very good agreement between the modelled major axis line-of-sight velocity diagrams and the observed optical L-O-S velocity curves for any pattern speed (see Fig. ??), probably due to the fact that in reality the inner bar rotates faster than the primary bar. The torques due to this decoupled bar are lower than those produced by an inner bar rotating at the same speed as the primary bar, leading to a less efficient flow of gas toward the centre (Combes 1994). This would be supported by the strong non-circular signature seen in the modelled velocity diagrams. One can also notice in the velocity curves that the slower bars show a more centrally concentrated gas distribution than the models with corotation closer to the bar ends. The major axis L-O-S velocity curves with $R_{\mathrm{CR}} / R_{\mathrm{bar}}=1.0,1.1$ and 1.2 seem to give an overall agreement of the rotation curve normalisation factor compared to the observations, favouring the adopted $M / L$ ratio calculated from population synthesis models.

\section{NGC 7267}

This is a four-armed barred $\mathrm{SB}(\mathrm{rs}) \mathrm{a}$ spiral at a distance of $44 \mathrm{Mpc}$. The bar is very bright and has complex structure in the continuum, in effect splitting at the ends. In $\mathrm{H} \alpha$, the dominant feature traces the bar. But there are bright complexes near the bar ends that do not correlate well with the continuum features. No clear ring patterns are evident in $\mathrm{H} \alpha$. Discrete HII regions and diffuse emission lie around the bar and in one of the outer arms (Crocker et al. 1996). There is an intense emission from the nucleus. The bar position angle is approximately at right angles to the line of nodes.

The pattern speeds explored in the simulations are shown in Table 5. The simulations fail to reach a stationary state for any of the pattern speeds (Fig. 15). Since no steady configuration was found, no comparison with the observed data was made. A detailed discussion of the possible reasons why the modelling fails is given in Sect. 11. One a priori reason why the gas fails to reach a steady state is the asymmetries in the light distribution. To test this possibility the potential was calculated from the bisymmetrised light distribution of NGC 7267 and the code was run with the standard parameters. Again, no steady state is reached for any of the pattern speeds.

\section{NGC 7483}

An outer and an inner ring are the main morphological features of the SABa galaxy NGC 7483 at a distance of $66 \mathrm{Mpc}$. It also seems to posses a nuclear ring perpendicular to the bar. There is no kinematic nor photometric study of this galaxy in the literature. The bar position angle is approximately at right angles to the line of nodes.

\subsection{Results}

The pattern speeds explored in the simulation are presented in Table 5. The gas density distribution clearly settles into a stationary configuration after three bar rotations, and for all pattern speeds a nuclear ring develops perpendicular to the bar (Fig. 18). From the frequency plot of the axisymmetric case this galaxy possesses two ILRs for all the pattern speeds explored (in the axisymmetric approximation). Although the general gas configuration remains stable, the nuclear ring does not remain steady and wobbles in time. Whether this ring is formed purely by particles confined to $x_{2}$ orbits will have to be checked through an orbit analysis. There is evidence from Fig. 19 of large velocity gradients, and the velocity vectors have different directions in the nuclear region indicating the presence of shocks. In fact, when one looks at the $B-I$ map (see Fig. 16) the presence of complicated dust lanes featuring several arm spiral structures in the nuclear region is evident.

The kinematics obtained through long slit spectroscopy at the $1.5 \mathrm{~m}$ at La Silla Observatory show a very complicated rotation curve (see Fig. 17); however a comparison of the modelled L-O-S velocity curve with the data shows a good agreement (see Fig. ??). As in the case of IC 5186, the amplitude of the rotation curve, fixed by the choice of $M / L$, agrees well with the observed rotation curve. The best fit is for a pattern speed of $27.50 \mathrm{~km} \mathrm{~s}^{-1} \mathrm{kpc}^{-1}$ corresponding to a corotation radius at the end of the bar (see Table 5).

A morphological comparison has been made for different pattern speeds following the method explained in Sect. 4.4 (see Fig. ??). Both the nuclear and the inner rings are present in the models. The size of these features when compared to the real galaxy suggests a fast rotating bar. On a larger scale, the models show a two armed spiral pattern. In the simulations some gas becomes trapped around the $L_{4,5}$ Lagrangian points with the maximum density enhancement offset from the zero velocity points. This effect can be seen in the density distribution (Fig. 19). 


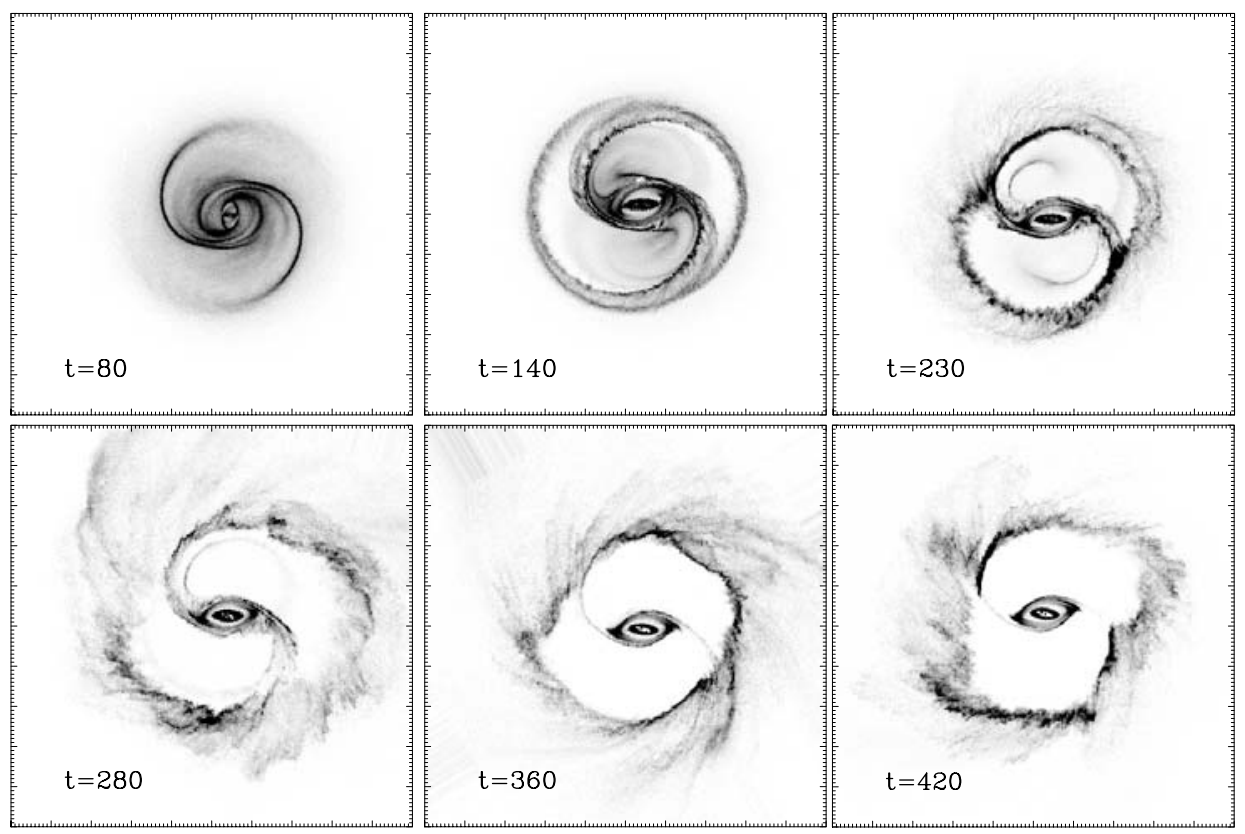

Fig. 12. Time evolution of the gas distribution in the NGC 5728 simulation with corotation at the end of the bar $\left(R_{\mathrm{CR}} / R_{\mathrm{bar}}=1.0\right)$; the bar pattern is rotating counter-clockwise. Each panel, from left to right and top to bottom, shows the distribution at different integration times, from 1 bar rotation to 6 bar rotation, and in the frame corotating with the bar. The non-axisymmetric component is fully grown after three bar rotations and the bar pattern is rotating counter-clockwise in the inertial frame. The size of the frames is $50 \mathrm{kpc}$ and time is in Myr.

\section{NGC 5505}

This is an isolated SBa galaxy at a distance of $57 \mathrm{Mpc}$. There is no kinematic nor photometric study of this galaxy in the literature. Morphologically it is characterised by a four-arm spiral structure and an inner ring. The bar is populated with HII regions and the nucleus shows intense emission in $\mathrm{H}_{\alpha}$. The bar position angle is at about $40^{\circ}$ with respect to the line of nodes.

The gas was left to evolve for 8 bar rotations. The pattern speeds explored in the simulations are shown in Table 5 . NGC 5505 is one of the two galaxies for which no steady state is reached for any of the pattern speeds explored. An example of the time evolution of the gas distribution of NGC 5505 is given in Fig. 22 for a $R_{\mathrm{CR}} / R_{\mathrm{bar}}=1.0$. The gas seems to dissipate energy through shocks and falls into the centre. In the final configuration, most of the gas is concentrated at the centre and some gas lies outside the OLR. Figure 22 suggests that these shocks are due to $x_{1}$ orbits that appear to loop over a large radial range. No ILRs are present according to the frequency diagram in the axisymmetric approximation, therefore the inner and nuclear ring do not seem to be associated to such a resonance and instead they are probably associated with the above $x_{1}$ orbits.

Since no steady configuration was found, no comparison with the observed data was carried out. However, to test whether a stationary state could be reached when including a rigid dark halo, runs with a dark halo were carried out. The results are presented in Sect. 10.2.

\section{Models with a dark halo component}

We ran simulations with a dark halo component to test the effect of a larger axisymmetric component on the gas distribution

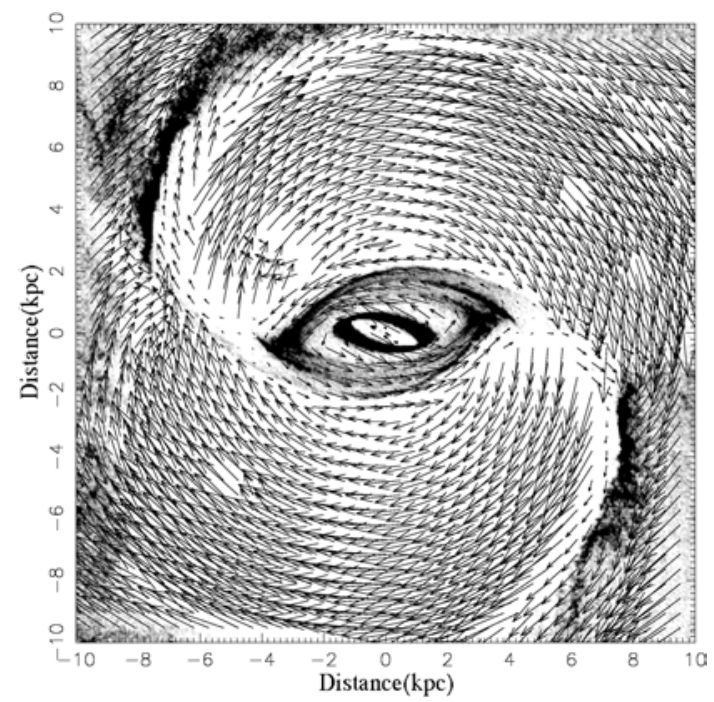

Fig. 14. The gas distribution and velocity field for the mass distribution derived from the composite $(H+I)$-band image of NGC 5728, at 6 bar rotations and for $R_{\mathrm{CR}} / R_{\mathrm{bar}}=1.0$. The length of the vectors is proportional to the velocity in the frame rotating with the bar.

and the kinematics of the modelled galaxies. Simulations were carried out on two of the five galaxies; IC 5186 and NGC 5505.

The tests on NGC 5505 were done to check whether introducing a dark halo could stabilise the gas flow. The dark halo was simulated by letting the bar grow to a percentage of its onset time and then continuing the simulations with this non-fully grown bar. This procedure mimics a lower stellar $M / L$, with the removed mass redistributed into an axisymmetric dark component (see Sect. 4). In this way we can test if the failure to reach a steady state was due to the fact that these galaxies do have 

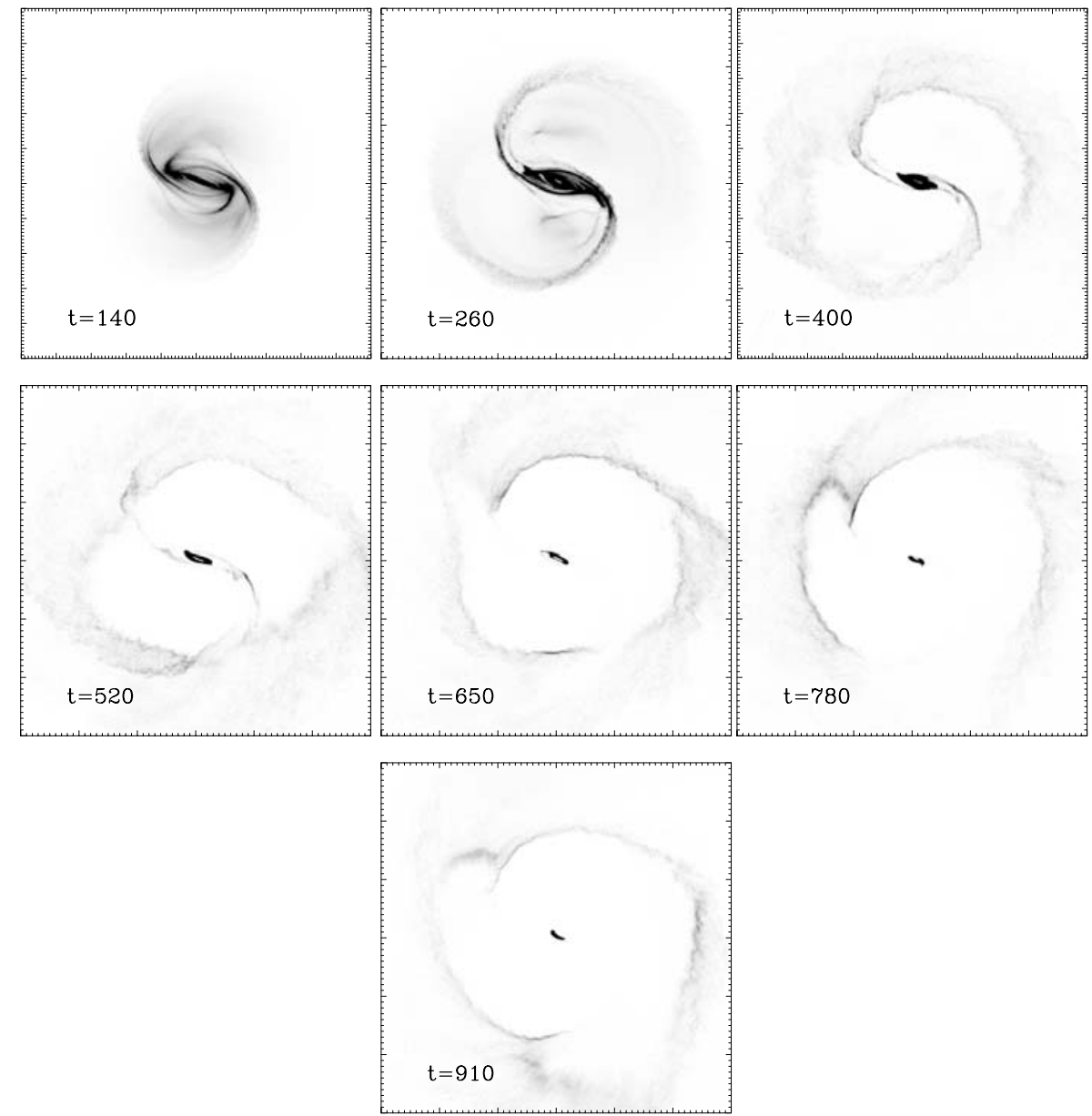

Fig. 15. Time evolution of the gas distribution in the NGC 7267 simulation with corotation at $1.0 \times$ the bar semi-major axis. Each panel, from left to right and top to bottom, shows the distribution at different integration times, from 1 bar rotation to 7 bar rotations, and in the frame corotating with the bar. The non-axisymmetric component is fully grown after three bar rotations and the bar pattern is rotating counterclockwise in the inertial frame. The size of the frames is $30 \mathrm{kpc}$ and time is in Myr. The gas does not reach a steady state and the inner feature shrinks further and further after every bar rotation.

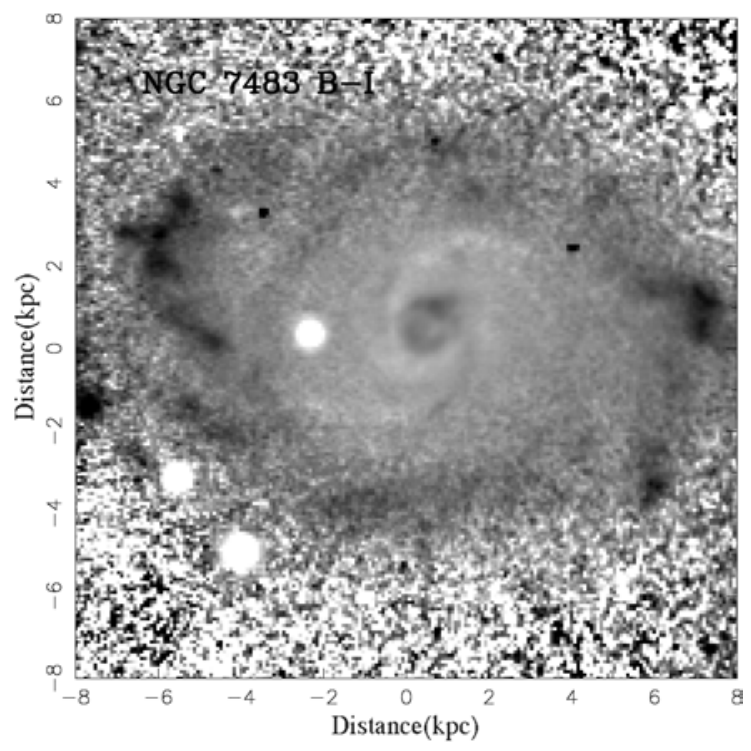

Fig. 16. $B-I$ map for NGC 7483 .

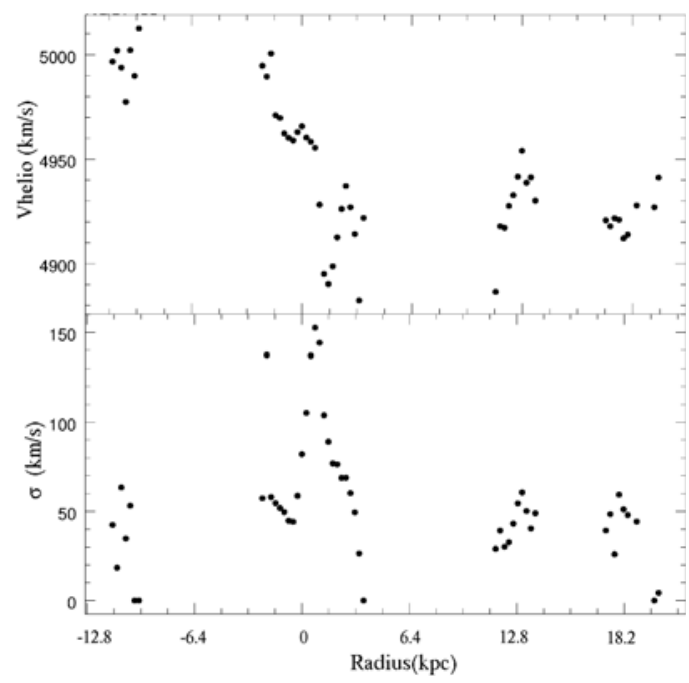

Fig. 17. Long-slit spectroscopic observations of the $\mathrm{H}_{\alpha}$ line of NGC 7483 with the slit oriented along the major axis. Notice how messy the rotation curve looks and compare it to the modelled rotation curve (Fig. ??). 


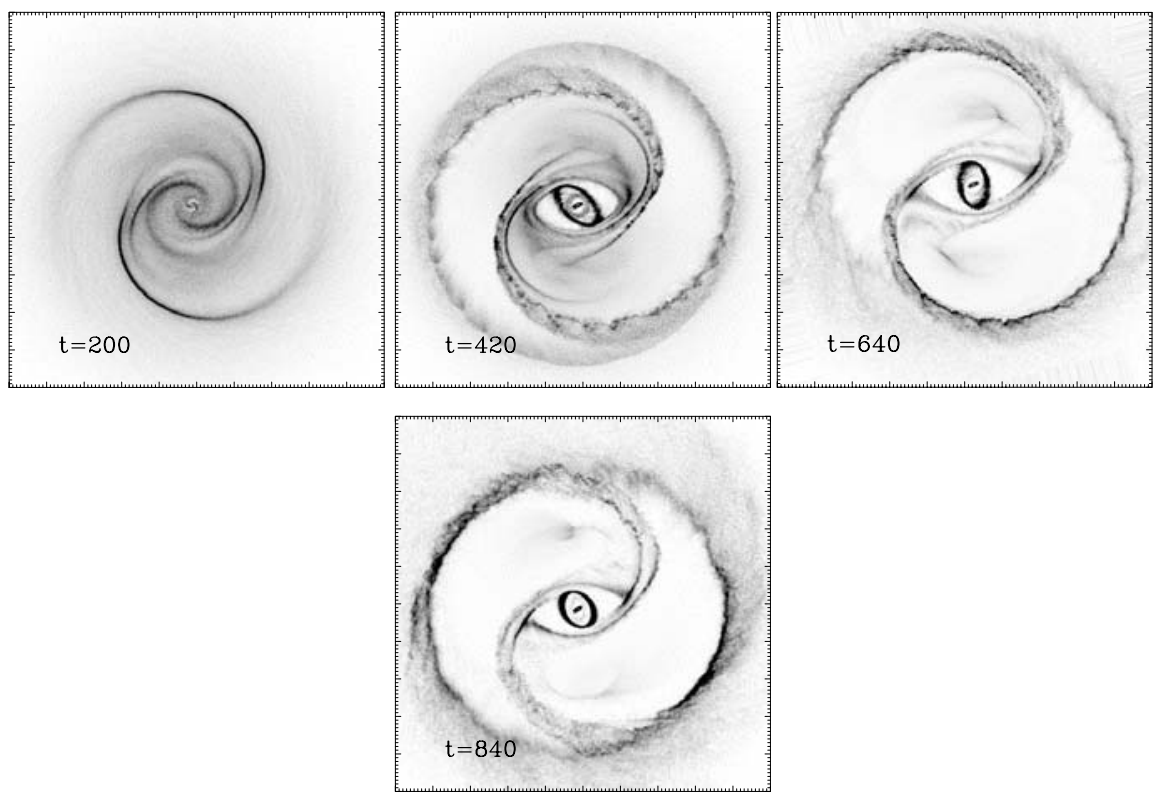

Fig. 18. Time evolution of the gas distribution in the NGC 7483 simulation with corotation at $1.0 \times$ the bar semi-major axis. The bar pattern is rotating counter-clockwise. Each panel, from left to right and top to bottom, shows the distribution at different integration times, from 1 bar rotation to 4 bar rotations, and in the frame corotating with the bar. The non-axisymmetric component is fully grown after three bar rotations and the bar pattern is rotating clockwise in the inertial frame. The size of the frames is $50 \mathrm{kpc}$ and time is in Myr.

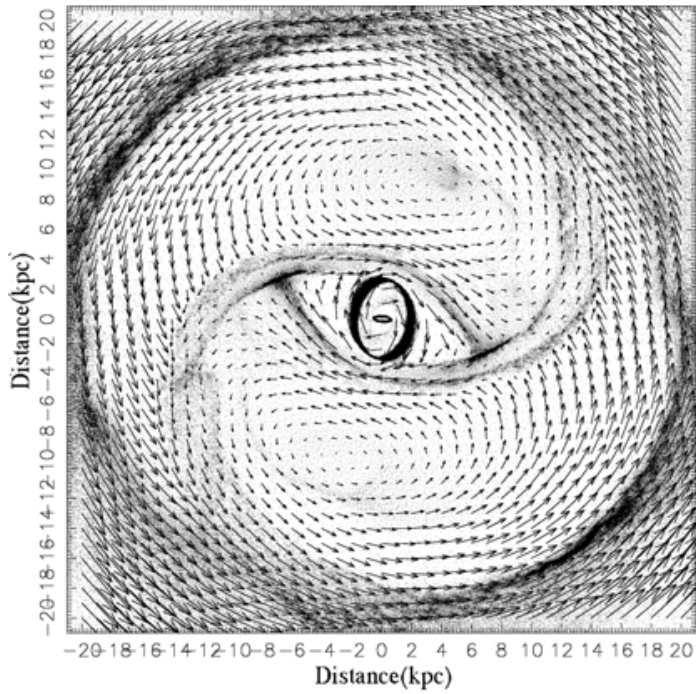

Fig. 19. The gas distribution and velocity field for the mass distribution derived from the composite $(H+I)$-band image of NGC 7483, at 4 bar rotations and for $R_{\mathrm{CR}} / R_{\mathrm{bar}}=1.0$. The length of the vectors is proportional to the velocity in the frame rotating with the bar.

a dark matter halo or because the modelling done here is not appropriate for this galaxy (e.g. because the stellar distribution is not in a sufficiently stationary state to allow a rigid potential approximation).

This test was also done for IC 5186 (presented in the first part of this section), the galaxy on which we have done most of the test simulations.

\subsection{IC 5186}

The parameter space covered by the simulations is the same as the one covered for the standard runs and three different dark halo contributions to the total mass were tried, namely $5 \%$, $20 \%$ and $40 \%$.

There is a clear change in the gas distribution when the dark halo contribution is increased, as one would expect from the increased presence of the axisymmetric component in the potential. The gas distribution is smoother and the inner ring seems to become more circular. Outside the corotation radius the distribution is very similar for all the dark halo mass percentages. One can see from Fig. ?? that there is no way to distinguish between the $0 \%$ and the $5 \%$ dark halo cases. However, for the cases with $20 \%$ and $40 \%$ dark halo mass, not only is the gas distribution very different to the heavy disk cases but it also diverges from the light distribution of the real galaxy significantly (see Figs. 1 and ??). The heavy disk models show more similarities with the deprojected $B$-band image than the light disk models.

The L-O-S velocity curves (see Fig. ??) show that the agreement with the observed data worsens for the case with $40 \%$ dark halo mass. The $\chi^{2} / N$ value is 2.66 for $5 \%$ dark halo mass, 2.69 for $20 \%$ dark halo mass and 3.79 for $40 \%$ dark halo mass. The $5 \%$ and $20 \%$ cases are almost indistinguishable from the case without a dark halo, whereas in the central 5 arcsec the model with $40 \%$ dark halo mass depart from the observed L-O-S velocity curve along the major axis.

The fact that a disk with $20 \%$ of the mass residing in the dark halo still gives a good agreement in the L-O-S velocity curve comparison agrees with the definition of maximum disk given at the introduction, where the stellar mass provides $85 \% \pm 10 \%$ of the total rotational support of the galaxy. 

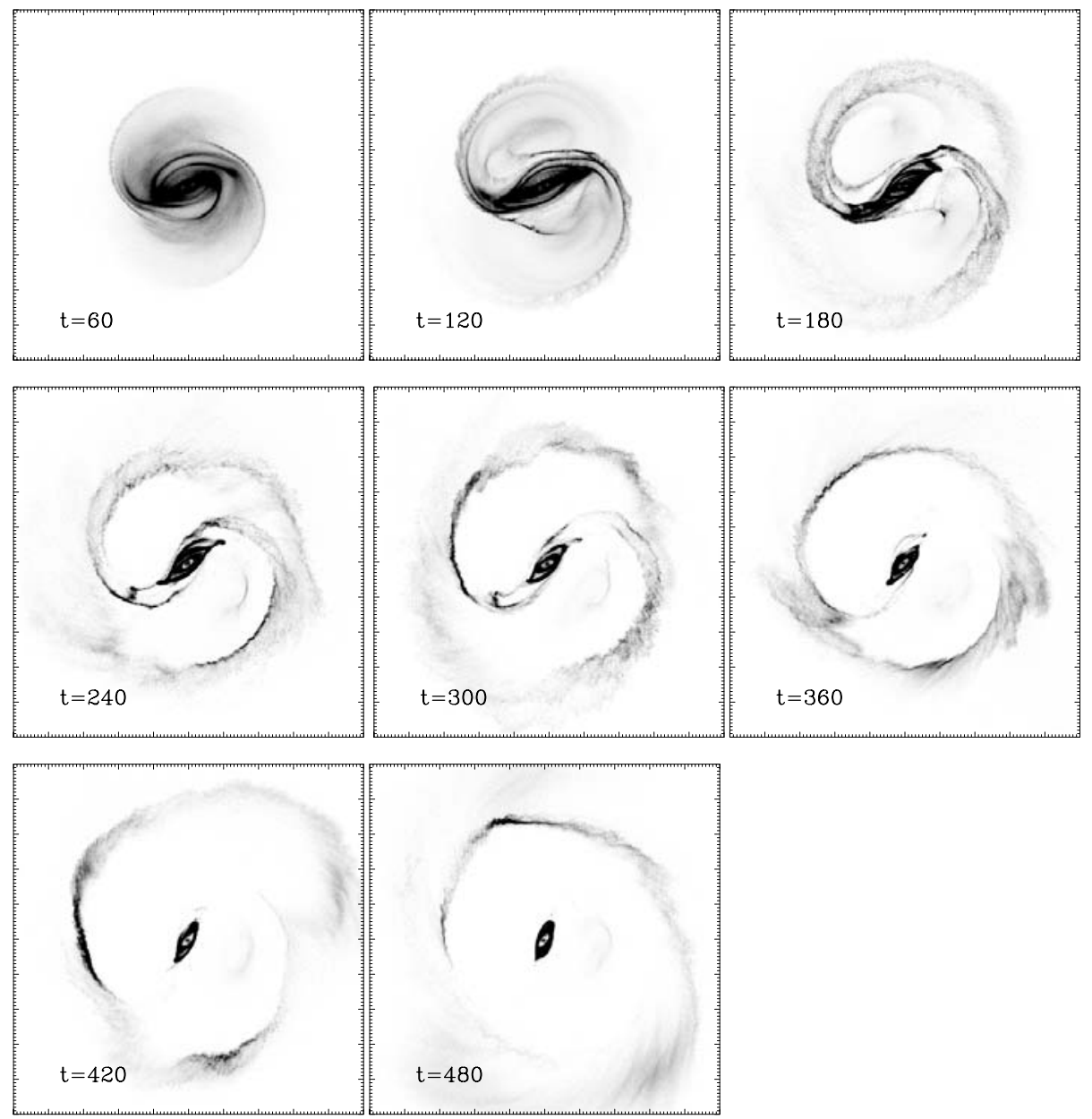

Fig. 22. Time evolution of the gas distribution in the NGC 5505 simulation with corotation at $1.4 \times$ the bar semi-major axis. Each panel, left to right and top to bottom, shows the distribution at different integration times, from 1 bar rotation to 8 bar rotations, and in the frame corotating with the bar. The non-axisymmetric component is fully grown after three bar rotations and the bar pattern is rotating counter-clockwise in the inertial frame. The size of the frames is $20 \mathrm{kpc}$ and time is in Myr.

Runs with different pattern speeds $\left(R_{\mathrm{CR}} / R_{\mathrm{bar}}=1.0\right.$ and $\left.R_{\mathrm{CR}} / R_{\mathrm{bar}}=1.6\right)$ for the different dark halo masses were also carried out. The $\chi^{2} / N$ values obtained for the kinematic comparisons were always larger than the ones obtained for $R_{\mathrm{CR}} / R_{\mathrm{bar}}=1.4$. The same behaviour as above is found for $R_{\mathrm{CR}} / R_{\mathrm{bar}}=1.0$. However, note that although the fit is not good for the case $R_{\mathrm{CR}} / R_{\mathrm{bar}}=1.6$ the $\chi^{2} / N$ diminishes as the dark halo mass increases.

\subsection{NGC 5505}

As seen in Sect. 9, no stationary state was reached in the simulations for NGC 5505 for any of the pattern speeds explored. The experience with all the simulations suggests that a slower bar would have produced an even more unstable result. To test if the gas could be stabilised with a dark halo component, simulations similar to those of the previous section on IC 5186 were carried out.

For a minimum dark halo mass of $20 \%$ of the total mass a stationary state is found for all pattern speeds. Figure ?? shows the L-O-S velocity curve for NGC 5505 in these cases. No good agreement with the data is found. Further discussion will be presented in Sect. 11.2.

The gas stabilises faster and evolves more slowly in the simulations with a dark matter halo but it is possible that with sufficient integration time the gas would have reached the same configuration as the simulations without a dark halo. For a dark halo mass of $5 \%$ of the total mass the simulation evolves in a similar fashion as in the case with no dark halo.

\section{Discussion}

\subsection{Why do some galaxies not reach a steady state?}

It is not clear why for some galaxies we obtained a stationary configuration while for others (NGC 5505 and NGC 7267) we could not. It is certainly related to the non-axisymmetric part of the potential, since the final gas distribution in the latter cases is due to a high degree of dissipation through the shocks. One would expect that in the cases where the galaxies show more star forming regions the $M / L$ calculation is less accurate. However, no trend is found with colour gradient. One of the galaxies with a large colour change in the bar and disk region 


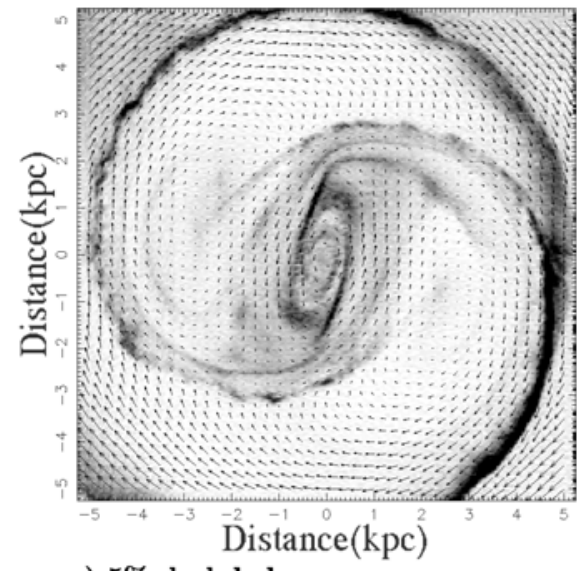

a) $5 \%$ dark halo mass

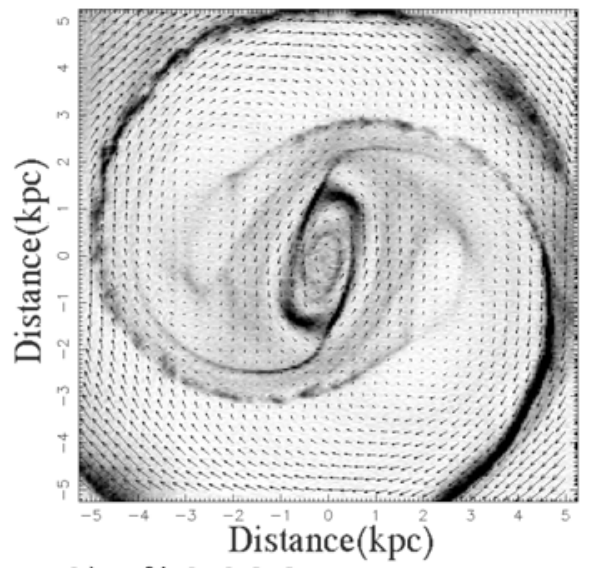

b) $20 \%$ dark halo mass

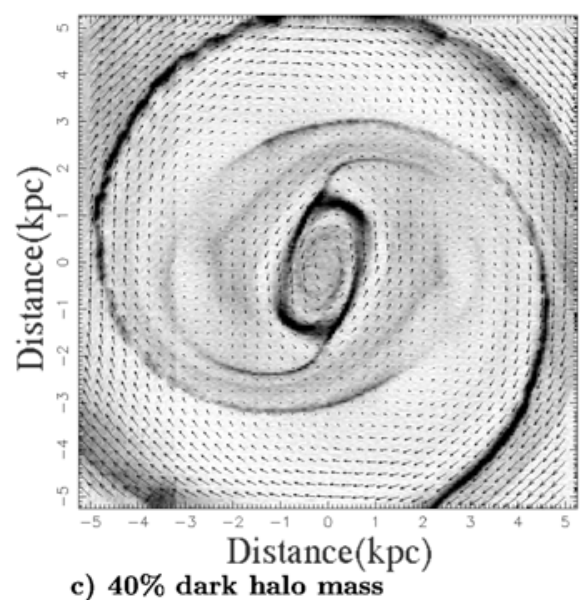

Fig. 23. The gas distribution and velocity field for the mass distributions of IC 5186 derived from the composite $(H+I)$-band image and with various dark halo mass fractions, at 8 bar rotations and for $R_{\mathrm{CR}} / R_{\mathrm{bar}}=1.4$. The length of the vectors is proportional to the velocity in the frame rotating with the bar.

is NGC 5505. However NGC 7483 also shows large colour differences across the bar region (Paper II) and NGC 7267 barely shows any change, i.e. has an almost constant $M / L$ value. See Paper II for the color maps of the individual galaxies.

To check the role played by light distribution asymmetries in the galaxies, runs with a bisymmetrised mass distribution were carried out for NGC 7267. Standard parameters were used for the simulations. No steady configuration was reached after integrating for 8 bar rotations.

Since one expects a larger degree of dissipation in stronger bars the bar strength in the sample galaxies was calculated to see if there was any correlation. To calculate the bar strength one has to take into account the radial and the tangential forces. The definition adopted here for the bar strength (Combes \& Sanders 1981; Block et al. 2002) is the maximum over $R$ of

$Q_{T}(R)=\frac{F_{T}^{\max }(R)}{F_{0}(R)}=\frac{\frac{1}{R}\left|\frac{\partial \Phi(R, \phi)}{\partial \phi}\right|_{\max }}{\frac{\mathrm{d} \Phi_{0}(R)}{\mathrm{d} R}}$

where $F_{T}^{\max }(R)$ is the azimuthal maximum of the absolute tangential force, $F_{0}(R)$ the mean axisymmetric radial force, $\Phi(R, \phi)$ the in-plane gravitational potential and $\Phi_{0}(R)$ its axisymmetric part.
Table 4. Bar strength for the modelled galaxies.

\begin{tabular}{clll}
\hline \hline Galaxy name & Bar strength & Bar class & $\begin{array}{l}\text { Steady pattern } \\
\text { achieved }\end{array}$ \\
\hline NGC 7483 & 0.34 & 3 & Yes \\
NGC 7267 & 0.56 & 6 & No \\
NGC 5728 & 0.16 & 2 & Yes \\
NGC 5505 & 0.44 & 4 & No \\
IC 5186 & 0.60 & 6 & Yes \\
\hline
\end{tabular}

This way of measuring the bar strength takes into account the axisymmetric disk in which the bar lies. Table 4 shows the results of the bar strength for the modelled galaxies, assuming the scale-heights adopted in the standard runs. In this table, the bar class refers to the bar strength classification introduced by Block et al. (2002), going from 1 to 6 , with class 6 representing the strongest bar. There seems to be no correlation between bar strength and the steadiness of the simulations. However, since the gas flow in the simulations for NGC 5505 stabilises after a rigid halo has been added, the steadiness of the simulations must be related to the level of dissipation due to the non-axisymmetric component. 


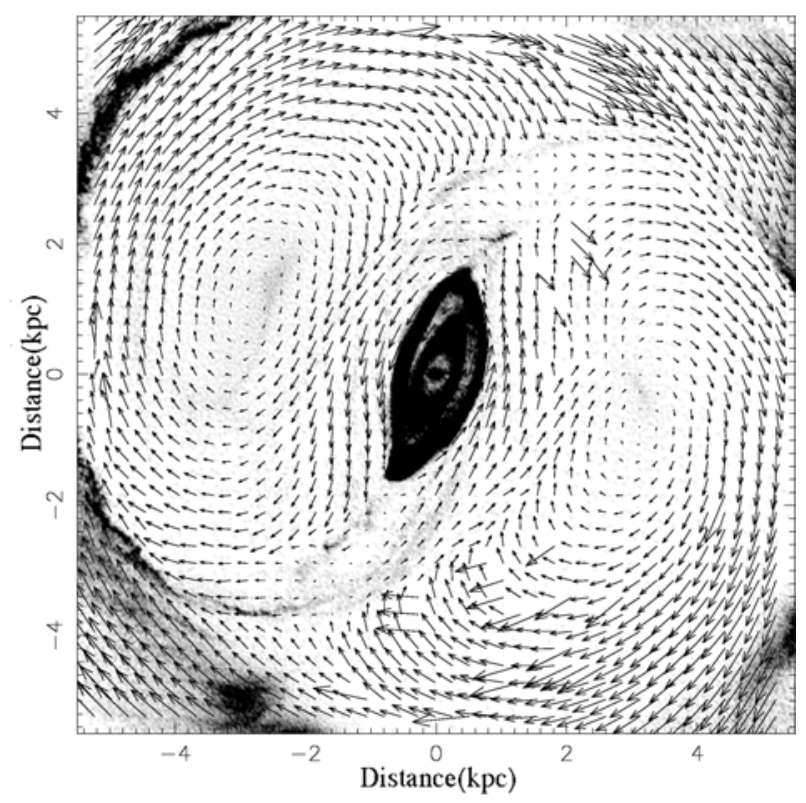

Fig. 26. The gas distribution and velocity field for the mass distribution of NGC 5505 derived from the composite $(H+I)$-band image and with $20 \%$ dark halo, at 8 bar rotations and for $R_{\mathrm{CR}} / R_{\mathrm{bar}}=1.0$. The length of the vectors is proportional to the velocity in the frame rotating with the bar.

\subsection{Does NGC 5505 need a dark halo?}

From the present data we can draw no conclusions about the dark matter component in the inner parts of NGC 5505, until we understand why the gas does not stabilise for NGC 5505 and NGC 7267. When one adds a dark halo component the gas settles faster; however, the L-O-S velocity curve does not give a good fit to the data. It is not possible to conclude anything about the need for a dark halo in NGC 5505. All that has been shown is the relationship between the fact that the gas does not reach a steady state and the addition of the dark halo component whose effect is to reduce the radial gas flow and smooth out the potential. The fact that the model velocities are too low in the central regions (see Fig. ??) may suggest that one should add a cuspy halo to increase the mass density in that region. But also adding a cusp reduces the non-circular motions and thus may lower the velocities in the L-O-S velocity curve, depending on the orientation of the slit relative to the bar. Note that, as pointed out by Athanassoula (2001), a heavy halo can enhance the bar growth by the exchange in the angular momentum between the two components, where the halo might take angular momentum from the bar. She argues that the formation of a bar causes the central concentration of the disk to increase so that the disk dominates more and more in the inner region and evolves into a maximum disk after the bar has grown. NGC 5505 may be an example of a strong bar embedded in a heavy halo.

\section{Conclusions}

The aim of the modelling is to see whether the mass distribution obtained from the light distribution in the $H$-band, together with the hydrodynamical simulations, can reproduce the observed rotation curves and the gas morphology of the inner regions of our galaxies. The $M / L$ is determined using population synthesis models. Since no additional mass component is added, the $M / L$ acts as a normalisation factor on the rotation curves. Therefore, testing the modelled L-O-S velocity curves is also a test of the population synthesis models. This turns out to be largely a test of the adopted IMF (see Paper II). However, we are not only testing the normalisation factor but also the shape of the central parts of the position-velocity diagram which in the case of barred galaxies has the characteristic imprints of the non-circular motions imposed by the bar. In this way we can test whether a dark halo component is needed to explain the observed kinematics in the inner regions of our galaxies.

The simulations presented here are compared to long-slit spectroscopy along the major axis, this could certainly produce degeneracies in the obtained parameters. These galaxies were too faint to obtain high spatial resolution HI maps and no optical integral field unit was available at the time. In order to provide better constraints, further comparison with $2 \mathrm{D}$ velocity fields will be necessary.

Hydrodynamical simulations are run for five of the sample galaxies, exploring different areas in parameter space. Different bar pattern speeds and different scale-heights are investigated. For three of the five galaxies a steady gas flow is reached after the non-axisymmetric component is fully grown. For two of these galaxies (NGC 7483 and IC 5186) the best fit pattern speed is found, both giving a fast bar (with $R_{\mathrm{CR}} / R_{\mathrm{bar}}=1.0$ for NGC 7483 and $R_{\mathrm{CR}} / R_{\text {bar }}=1.4$ for IC 5186). For the third one, NGC 5728, the models do not fit well the observed data, possibly due to the presence of a secondary bar decoupled from the primary bar. However, the $M / L$ ratio adopted seems to give the right normalization factor. For the other two galaxies (NGC 7267 and NGC 5505), no steady gas flow is achieved: the dissipative shocks make the gas particles fall toward the centre, leaving most of the gas at the centre and in an outer 
Table 5. Model parameters for the modelled galaxies.

\begin{tabular}{llccc}
\hline \hline Galaxy name & $R_{\mathrm{CR}} / R_{\text {bar }}$ & $\begin{array}{c}\text { Corotation } \\
(\mathrm{kpc})\end{array}$ & $\begin{array}{c}\Omega_{\mathrm{b}} \\
\left(\mathrm{km} \mathrm{s}^{-1} \mathrm{kpc}^{-1}\right)\end{array}$ & $\chi^{2} / N$ \\
\hline IC 5186 & 1.00 & 2.2 & 86.2 & 6.69 \\
& 1.10 & 2.4 & 79.6 & 5.94 \\
& 1.20 & 2.6 & 73.6 & 2.36 \\
& 1.30 & 2.8 & 68.3 & 2.55 \\
& 1.40 & 3.0 & 63.4 & 2.25 \\
& 1.60 & 3.5 & 55.2 & 3.56 \\
\hline NGC 5728 & 1.00 & 5.1 & 81.9 & \\
& 1.10 & 5.6 & 73.3 & \\
& 1.20 & 6.2 & 66.5 & \\
& 1.30 & 6.7 & 60.9 & \\
& 1.40 & 7.2 & 56.2 & \\
& 1.60 & 8.2 & 48.9 & \\
\hline NGC 7267 & 1.00 & 4.5 & 44.8 & \\
& 1.10 & 4.9 & 40.7 & \\
& 1.20 & 5.4 & 37.2 & \\
& 1.30 & 5.8 & 34.1 & \\
& 1.40 & 6.3 & 31.4 & \\
& 1.60 & 7.2 & 27.1 & \\
\hline NGC 5505 & 1.00 & 3.3 & 99.3 & \\
& 1.10 & 3.7 & 91.2 & \\
& 1.20 & 4.0 & 83.2 & \\
& 1.30 & 4.3 & 75.8 & \\
& 1.40 & 4.6 & 69.3 & \\
& 1.60 & 5.3 & 59.1 & \\
& 1.10 & 10.5 & 24.8 & 2.25 \\
& 1.20 & 11.5 & 22.7 & 2.04 \\
& 1.30 & 12.4 & 20.8 & 2.05 \\
& 1.40 & 13.4 & 19.2 & 1.65 \\
& 1.60 & 15.3 & 16.3 & 1.87 \\
\hline
\end{tabular}

ring probably associated to the OLR. However, when $20 \%$ of the non-axisymmetric mass component is replaced by an axisymmetric dark halo component the gas manages to reach a steady state.The simulations with short scale-heights do not give a good fit for any of the pattern speeds. However, simulations run with higher scale-heights give better fits, although the scale-heights are unrealistic for real galaxies.

Simulations were run for potentials obtained from the $I$-band light distribution and from the composite of a $H$-band image at the centre and $I$-band image in the outer isophotes. Such models show differences in the gas distribution resulting from the higher dust absorption in the central regions of the $I$-band image. For example, the simulations in the potential derived from the $I$-band image alone show a substantially more pronounced nuclear ring, whereas the simulations in the potential derived from the composite $(H+I)$ image seem to have more gas trapped around the $L_{4 / 5}$ Lagrangian points. However, only small differences between the two simulations are found in the kinematics along the major axis.
We tested the effect of a dark halo component in the simulations, by converting part of the visible mass inferred from the population synthesis models into an axisymmetric component, simulating the effect of a lower stellar $M / L$ and rigid halo. The fit to the observations becomes significantly worse.

The $M / L$ ratios are obtained from the population synthesis models give the right normalisation factor to match the modelled position-velocity diagrams to the observed rotation curve, indicating that the population synthesis models do give realistic $M / L_{H}$ values. For the above galaxies in which full comparison of the rotation curve has been done, not only the normalisation, but the shape of the rotation curve agrees very well with the data, even in the case of NGC 7483 which possesses a very complicated and messy rotation curve.

Acknowledgements. We would like to thank the APAC super computer facility for providing the computing time and facilities that made this work possible. This work was supported by the Australian National University through an ANU Ph.D. grant. We would also like to thank the external referees of the Ph.D. Thesis associated to this paper for their useful comments and Hervé Wozniak for his constructive criticism; which helped in the improvement of this manuscript.

\section{References}

Athanassoula, E. 1992, MNRAS, 259, 345

Athanassoula, E. 2002, ApJ, 569, 83

Benz, W. 1990, in The Numerical Modelling of Nonlinear Stellar Pulsations Problems and Prospects (Kluwer), NATO ASI Ser. C, 302, 269

Block, D., Buta, R., Puerari, I., et al. 2002, in The Dynamics, Structure and History of Galaxies, ASP Conf. Proc., 273, 97

Carter, B. 1995, Ap\&SS, 230, 163

Combes, F. 1994, in Mass-Transfer Induced Activity in Galaxies, ed. I. Shlosman, 170

Combes, F., \& Leon, S. 2002, in SF2A-2002: Semaine de l'Astrophysique Française, ed. F. Combes, \& D. Barret (Les Ulis: EDP Sciences), 379, 403

Combes, F., \& Sanders, R. 1981, A\&A, 96, 164

Courteau, S., \& Rix, H.-W. 1996, ApJ, 513, 561

Crocker, D., Baugus, P., \& Buta, R. 1996, in Barred galaxies, ed. R. Buta, D. Crocker, \& B. Elmegreen, PASP, 91, 80

de Jong, R. S. 1996, A\&A, 313, 45

Debattista, V., \& Sellwood, J. 1998, ApJ, 493, 5

Freeman, K. 1992, IAUS, 149, 65

Freudenreich, H. 1998, ApJ, 492, 495

Fux, R. 1997, Ph.D. Thesis, Geneva University

Fux, R. 1999, A\&A, 345, 787

Kregel, M., der Kruit, P., \& de Grijs, R. 2002, MNRAS, 334, 646

Landolt, A. 1992, AJ, 107, 372

Pérez, I. 2003, Ph.D. Thesis, Australian National University

Persson, S., Murphy, D., Krzeminski, W., Roth, M., \& Rieke, M. 1998, AJ, 116, 2475

Pfenniger, D., \& Friedli, D. 1993, A\&A, 270, 561

Regan, M., \& Teuben, P. 2003, ApJ, 582, 723

Sackett, P. 1997, ApJ, 483, 103

Salucci, P., \& Persic, M. 1999, A\&A, 351, 442

Tremaine, S., \& Ostriker, J. 1999, MNRAS, 306, 662

Weinberg, M. 1985, MNRAS, 213, 451

Weiner, B., Sellwood, J., \& Williams, T. 2001, ApJ, 546, 931

Wozniak, H., Friedli, D., Martinet, L., Martin, P., \& Bratschi, P. 1995, A\&A, 111, 115 